\title{
Stromal cells in the tumor microenvironment promote the progression of oral squamous cell carcinoma
}

\author{
QIUSHENG SHAN $^{1,2}$, KIYOFUMI TAKABATAKE $^{1}$, HARUKA OMORI $^{1}$, \\ HOTAKA KAWAI $^{1}$, MAY WATHONE OO ${ }^{1}$, KEISUKE NAKANO ${ }^{1}$, \\ SOICHIRO IBARAGI ${ }^{2}$, AKIRA SASAKI ${ }^{2}$ and HITOSHI NAGATSUKA ${ }^{1}$ \\ Departments of ${ }^{1}$ Oral Pathology and Medicine, and ${ }^{2}$ Oral and Maxillofacial Surgery, \\ Okayama University Graduate School of Medicine, Dentistry, and \\ Pharmaceutical Sciences, Kita-ku, Okayama 700-8525, Japan
}

Received May 28, 2021; Accepted July 22, 2021

DOI: $10.3892 /$ ijo.2021.5252

\begin{abstract}
The stromal cells in the tumor microenvironment (TME) can influence the progression of multiple types of cancer; however, data on oral squamous cell carcinoma (OSCC) are limited. In the present study, the effects of verrucous squamous cell carcinoma-associated stromal cells (VSCC-SCs), squamous cell carcinoma-associated stromal cells (SCC-SCs) and human dermal fibroblasts (HDFs) on the tumor nest formation, proliferation, invasion and migration of HSC-3 cells were examined in vitro using Giemsa staining, MTS, and Transwell (invasion and migration) assays, respectively. The results revealed that both the VSCC-SCs and SCC-SCs inhibited the tumor nest formation, and promoted the proliferation, invasion and migration of OSCC cells in vitro. Furthermore, the effects of VSCC-SCs, SCC-SCs and HDFs on the differentiation, proliferation, invasion and migration of OSCC cells in vivo were evaluated by hematoxylin and eosin staining, tartrate-resistant acid phosphatase staining, immunohistochemistry and double-fluorescent immunohistochemical staining, respectively. The results demonstrated that the VSCC-SCs promoted the differentiation, proliferation, invasion and migration of OSCC cells, while the SCC-SCs inhibited the differentiation, and promoted the proliferation, invasion and migration of OSCC cells in vivo. Finally, microarray data were used to predict genes in VSCC-SCs and SCC-SCs that may influence the progression of OSCC, and those with potential to influence the differential effects of VSCC-SCs and SCC-SCs on the differentiation of OSCC. It was found that $\mathrm{C}-\mathrm{X}$-C motif chemokine ligand (CXCL)8, mitogen-activated protein kinase 3
\end{abstract}

Correspondence to: Dr Kiyofumi Takabatake, Department of Oral Pathology and Medicine, Okayama University Graduate School of Medicine, Dentistry, and Pharmaceutical Sciences, 2-5-1 Shikata-cho, Kita-ku, Okayama 700-8525, Japan

E-mail: gmd422094@s.okayama-u.ac.jp

Key words: oral squamous cell carcinoma, stromal cells, differentiation, proliferation, invasion, migration, microarray
(MAPK3), phosphatidylinositol-4,5-bisphosphate 3-kinase catalytic subunit alpha (PIK3CA), C-X-C motif chemokine ligand 1 (CXCL1) and C-C motif chemokine ligand 2 (CCL2) may be involved in the crosstalk between VSCC-SCs, SCC-SCs and OSCC cells, which regulates the progression of OSCC. Intercellular adhesion molecule 1 (ICAM1), interleukin (IL)1B, Fos proto-oncogene, AP-1 transcription factor subunit (FOS), bone morphogenetic protein 4 (BMP4), insulin (INS) and nerve growth factor (NGF) may be responsible for the differential effects of VSCC-SCs and SCC-SCs on the differentiation of OSCC. On the whole, the present study demonstrates that both VSCC-SCs and SCC-SCs may promote the progression of OSCC, and SCC-SCs were found to exert a more prominent promoting effect; this may represent a potential regulatory mechanism for the progression of OSCC.

\section{Introduction}

Oral squamous cell carcinoma (OSCC) is the most common malignant tumor of the head and neck region, which is caused by smoking, alcohol consumption and viral infections. The main treatment for OSCC consists of surgery combined with chemotherapy and radiotherapy $(1,2)$. Despite advancements being made in the diagnosis and treatment of the disease, the 5-year survival rate of patients with OSCC remains low at $50 \%$, largely due to tumor recurrence and metastasis $(3,4)$. Therefore, there is a need to further investigate the regulatory mechanisms underlying OSCC differentiation, proliferation, invasion and metastasis in order to improve the poor prognosis of patients with OSCC.

Crosstalk between the tumor microenvironment (TME) and tumors can promote cancer progression $(5,6)$. Stromal cells, which are the main component of the TME, can influence the proliferation, invasion and metastasis of cancers (7). Stromal cells consist of cancer-associated fibroblasts (CAFs), tumor-associated macrophages (TAMs) and infiltrating immune cells, which can enhance cancer cell proliferation and metastasis by forming a supportive environment for angiogenesis, invasion and metastasis (8-10). A previous study suggested that the interaction between stromal cells and the epithelium promotes the progression of breast cancer (11). In addition, the 
expression of programmed death-ligand 1 (PD-L1) on stromal cells promotes the progression of colon cancer by suppressing $\mathrm{CD}^{+} \mathrm{T}$-cell immune responses (12). Mesenchymal stromal cells can also promote cancer metastasis by enhancing epithelial-to-mesenchymal transition (EMT) (13). Therefore, stromal cells may influence the progression of multiple cancer types by interacting with cancer cells; however, data on the influence of stromal cells in OSCC are limited.

Clinically, various subtypes of OSCC exist, including invasive carcinoma and verrucous carcinoma. Differences in the invasive ability of these subtypes results in marked differences in prognosis. The endophytic type (ED-type) OSCC can invade and occasionally metastasize. Conversely, the exophytic type (EX-type) OSCC, such as verrucous OSCC, presents an outward growth, does not invade the subepithelial connective tissue, does not metastasize and is therefore associated with a relatively good prognosis (14-16). However, to the best of our knowledge, no studies available to date have examined the mechanisms through which differences in these subtypes affect the tumor stroma. Therefore, it was hypothesized that different subtypes of stromal cells in the TME differentially affect the progression of OSCC. To examine this hypothesis, in the present study, the moderately differentiated human oral cancer cell line, HSC-3, was used as a cell model and verrucous squamous cell carcinoma-associated stromal cells (VSCC-SCs; derived from EX-type OSCC stroma) and squamous cell carcinoma-associated stromal cells (SCC-SCs; derived from ED-type OSCC stroma) were extracted from patients with OSCC to examine the effects of different stromal cells subtypes in the TME on the progression of OSCC. Furthermore, microarray data and bioinformatics analyses were used to elucidate the potential mechanisms underlying the differential effects of stromal cell subtypes on the progression of OSCC. These findings may highlight a potential regulatory mechanism underlying the progression of OSCC.

\section{Materials and methods}

Cells and cell culture. The moderately differentiated human oral cancer cell line, HSC-3 was purchased from the Cell Bank of the Japanese Collection of Research Bioresources (JCRB). Human dermal fibroblasts (HDFs) were purchased from Lonza Group, Ltd. VSCC-SCs and SCC-SCs were extracted from surgical operative tissues at the Department of Oral and Maxillofacial Surgery at Okayama University. The VSCC tissues and SCC tissues were obtained from each 1 patient, respectively to separate the stromal cells and generated the cell culture. Sections of fresh oral squamous carcinoma tissue $\left(1 \mathrm{~mm}^{3}\right)$ were washed several times with $\alpha$-Modified Eagle's medium ( $\alpha$-MEM) (Life Technologies; Thermo Fisher Scientific, Inc.) containing antibiotic-antimycotic (Life Technologies; Thermo Fisher Scientific, Inc.) and then minced. The tissues were then treated with $\alpha$-MEM containing $1 \mathrm{mg} / \mathrm{ml}$ collagenase II (Invitrogen; Thermo Fisher Scientific, Inc.) and dispase (Invitrogen; Thermo Fisher Scientific, Inc.) for $2 \mathrm{~h}$ at $37^{\circ} \mathrm{C}$ with shaking (200 rpm). The released cells were centrifuged for $5 \mathrm{~min}$ at $111.8 \mathrm{xg}$ at room temperature, suspended in $\alpha$-MEM containing 10\% FBS (Biowest), filtered through a cell strainer (100 $\mu \mathrm{m}, \mathrm{BD}$ Falcon; BD Biosciences), plated in a tissue culture flask and incubated at $37^{\circ} \mathrm{C}$ in
$5 \% \mathrm{CO}_{2}$. After 1 week, the stromal cells were separated by Accutase (Invitrogen; Thermo Fisher Scientific, Inc.) based on the differential adhesion of epithelial and stromal cells. These stromal cells were labeled VSCC-SCs (derived from EX-type OSCC stroma) and SCC-SCs (derived from ED-type OSCC stroma) (17). The HSC-3 cells, HDFs, VSCC-SCs and SCC-SCs were maintained in $\alpha$-MEM (Life Technologies; Thermo Fisher Scientific, Inc.) supplemented with 10\% FBS and $1 \%$ antimycotic-antibiotic (Life Technologies; Thermo Fisher Scientific, Inc.) at $37^{\circ} \mathrm{C}$ in a humidified atmosphere of $5 \% \mathrm{CO}_{2}$ and $95 \%$ air. The present study was approved by the Ethics Committee of Okayama University (project identification code: 1703-042-001). Informed consent was obtained from all patients.

Giemsa staining. VSCC-SCs, SCC-SCs, HDFs and HSC-3 cells were digested with Accutase and EDTA (Life Technologies; Thermo Fisher Scientific, Inc.), and centrifuged when the density approached $90 \%$. The VSCC-SCs, SCC-SCs and HDFs were then mixed with the HSC-3 cells at a 3:1 ratio. The cells were seeded into small dishes (Violamo, $35 \times 12 \mathrm{~mm}$ ) with a slide (Matsunami, $22 \times 22 \mathrm{~mm}$ ) at a density of $4 \times 10^{5} / \mathrm{dish}$. Following incubation for 1 day $\left(37^{\circ} \mathrm{C}\right)$, the attached slides were stained using the Giemsa Staining kit (Diff-Quick; Nanjing Jiancheng Bioengineering Institute). The slides were first washed with double-distilled water (DDW) and fixed with Diff-Quik Fixative provided with the Giemsa staining kit for $2 \mathrm{~min}$ at room temperature respectively. The slides were then stained with Diff-Quik Solution I and Diff-Quik Solution II provided with the Giemsa staining kit for $2 \mathrm{~min}$ at room temperature. Finally, the stained cells were photographed under a bright field microscope (x4, x10, x20 and $x 40$ magnification; BX51, Olympus Corporation). Independent experiments were repeated three times.

MTS assay. The VSCC-SCs, SCC-SCs, HDFs and HSC-3 cells were digested with Accutase and EDTA, and centrifuged when the density approached $90 \%$. The VSCC-SCs, SCC-SCs and HDFs were then mixed with HSC-3 at a 3:1 ratio. The cells were seeded into 96-wells plates at a density of 2,000 cells/well. Following incubation for 1,3 and 5 days, respectively $\left(37^{\circ} \mathrm{C}\right), 20 \mu \mathrm{l}$ of MTS regent were added to each well and the cells were then incubated for a further $4 \mathrm{~h}\left(37^{\circ} \mathrm{C}\right)$. The absorbance of each well was measured at $490 \mathrm{~nm}$ using an enzyme-linked immunosorbent assay reader (SH-1000 Lab). The OD values of the HSC-3 + VSCC-SCs/SCC-SCs/HDFs were compared with those of the HSC-3 (2,000 cells) cells and VSCC-SCs/SCC-SCs/HDFs $(2,000$ cells $)$ cells to determine the effects on the proliferation between the HSC-3 cells and VSCC-SCs/SCC-SCs/HDFs as follows: A promotion of cell proliferation was considered when the OD value of the HSC-3 + VSCC-SCs/SCC-SCs/HDFs mixed group was greater than that of the HSC-3 (2,000 cells) and VSCC-SCs/SCC-SCs/HDFs (2,000 cells) groups. An inhibition of cell proliferation was considered when the OD value of the HSC-3 + VSCC-SCs/SCC-SCs/HDFs mixed group was less than that of the HSC-3 (2,000 cells) and VSCC-SCs/SCC-SCs/HDFs (2,000 cells) groups. No effect on cell proliferation was considered to have occurred when the OD value of the HSC-3 + VSCC-SCs/SCC-SCs/HDFs 
mixed group was intermediate between that of the HSC-3 (2,000 cells) and VSCC-SCs/SCC-SCs/HDFs (2,000 cells) groups. Independent experiments were repeated three times.

Immunohistochemistry (IHC; Ki-67 labeling index). The slides were washed three times with TBS and fixed with $4 \%$ paraformaldehyde for $15 \mathrm{~min}$. Endogenous peroxidase activity was blocked by incubating the slides in methanol containing $0.3 \%$ $\mathrm{H}_{2} \mathrm{O}_{2}$ for $30 \mathrm{~min}$ and blocking liquid (DS Pharma Biomedical Co., Ltd.) for $20 \mathrm{~min}$. The primary antibody, mouse anti-Ki-67 (M7240, MIB1, 1:50; Dako; Agilent Technologies, Inc.) was added and slides were incubated for $2 \mathrm{~h}$ at room temperature. After washing three times with TBS, the slides were incubated with secondary antibody avidin-biotin complexes [PK-6102, mouse ABC kit; blocking serum (normal serum, diluted with TBS): 1:75; biotinylated secondary antibody (diluted with normal serum): 1:200; reagent A (Avidin, ABC Elite) and reagent $\mathrm{B}$ (Biotinylated $\mathrm{HRP}, \mathrm{ABC}$ Elite, diluted with TBS): 1:55; Vector Laboratories, Inc.] for $1 \mathrm{~h}$ at room temperature. Following visualization with a mixed solution of diaminobenzidine (DAB) $/ \mathrm{H}_{2} \mathrm{O}_{2}$ (histofine DAB substrate; Nichirei Biosciences Inc.), the stained slides were photographed under a bright field microscope (x4, x10, x20 and $\times 40$ magnification, BX51, Olympus Corporation). A total of five images ( $\mathrm{x} 40$ magnification) were randomly obtained and used to determine positive cell counts using ImageJ software (V1.51j8, National Institutes of Health). The percentage of positive Ki-67 cells was calculated as the number of positive $\mathrm{Ki}-67$ cancer cells/the number of all cancer cells x $100 \%$. Independent experiments were repeated three times.

Transwell invasion and migration assays, and immunofluorescence (IF). The VSCC-SCs, SCC-SCs, HDFs and HSC-3 cells were digested with Accutase and EDTA, and centrifuged for $5 \mathrm{~min}$ at $111.8 \mathrm{x} \mathrm{g}$ at room temperature when the density approached $90 \%$. The VSCC-SCs, SCC-SCs and HDFs were then mixed with HSC-3 cells at a 3:1 ratio in $\alpha$-MEM without FBS. For the Transwell invasion assay, the cells were seeded in the upper chamber of $8-\mu \mathrm{m}$ Transwell filters pre-coated with Matrigel in 24-well plates (Corning BioCoat Matrigel Invasion Chamber kit; BD Biosciences) at a density of 4x10 $/ 500 \mu \mathrm{l}$. Subsequently, $\alpha$-MEM $(500 \mu \mathrm{l})$ containing $10 \% \mathrm{FBS}$ was added to the bottom chamber. For the Transwell migration assay, the cells were seeded into the upper chamber of $8-\mu \mathrm{m}$ Transwell filters without Matrigel in 24-well plates (Corning, Falcon cell culture inserts; BD Biosciences) at a density of $2 \times 10^{4} / 500 \mu 1$. Subsequently, $\alpha$-MEM $(500 \mu \mathrm{l})$ containing $10 \% \mathrm{FBS}$ was added to the bottom chamber. Following 1 day of incubation $\left(37^{\circ} \mathrm{C}\right)$, the cells in upper chamber were removed using a cotton swab, and the membrane was trimmed in the upper chamber. After washing with TBS three times ( $5 \mathrm{~min}$ each), the cells were fixed with $4 \%$ paraformaldehyde for $15 \mathrm{~min}$ and blocked with blocking liquid (DS Pharma Biomedical Co., Ltd.) for $20 \mathrm{~min}$. The primary antibodies, rabbit anti-vimentin (ab16700, SP20, 1:200, Abcam) and mouse anti-pan cytokeratin (IS053, AE1/3, Abcam) were added and the cells were then incubated for $1 \mathrm{~h}$ at room temperature. After washing three times with TBS, the secondary antibodies, anti-mouse IgG Alexa Fluor 488 (A21441, 1:200, Life Technologies; Thermo Fisher Scientific, Inc.) and anti-rabbit IgG Alexa Fluor 568 (A10042, 1:200, Life
Technologies; Thermo Fisher Scientific, Inc.) were added and the cells were incubated for $1 \mathrm{~h}$ at room temperature without light. After washing with TBS and DDW three times, the samples were stained with $0.2 \mathrm{~g} / \mathrm{ml}$ 40,6-diamidino-2-phenylindole (DAPI; Dojindo Molecular Technologies, Inc.). The stained cells were photographed using a fluorescence microscope (x5 and x10 magnification, BZ-8000; Keyence). Independent experiments were repeated three times, and the data were analyzed using ImageJ software (V1.51j8).

Animal experiments. All animal experiments were conducted according to the relevant guidelines and regulations, and were approved by the institutional committees at Okayama University (OKU-2017406). Following intraperitoneal anesthesia with ketamine hydrochloride $(75 \mathrm{mg} / \mathrm{kg}$ body weight) and medetomidine hydrochloride $(0.5 \mathrm{mg} / \mathrm{kg}$ body weight $)$, $200 \mu \mathrm{l}$ mixed cells including HSC-3 $\left(1 \times 10^{6}, 100 \mu \mathrm{l}\right)$ and stromal cells (VSCC-SCs, SCC-SCs and HDFs, 3x10 ${ }^{6}, 100 \mu \mathrm{l}$ ) were injected into the central region of the top of the head (in the lamina propria) of $12 \mathrm{BALB} / \mathrm{c}$ nu-nu mice (4-week-old healthy females) obtained from SHIMIZU Laboratory Supplies Co., Ltd, as previously described $(18,19)$. All mice were reared in an animal room at a temperature of $25^{\circ} \mathrm{C}$ and $50-60 \%$ humidity under a 12-h light/dark cycle. All mice were allowed free access to food and water. Finally, atipamezole hydrochloride ( $1 \mathrm{mg} / \mathrm{kg}$ body weight) was subcutaneously injected into the abdominal cavity to reverse anesthesia. The experimental animals were divided into the HSC-3, HSC-3 + VSCC-SCs, HSC-3 + SCC-SCs and HSC-3 + HDFs groups and each group consisted of three mice.

Hematoxylin and eosin $(H \& E)$ staining. After 4 weeks, all mice were sacrificed by isoflurane excess inhalation anesthesia (concentration $>5 \%$ ). Cardiac arrest was then verified by pulse palpation followed by the dislocation of the cervical spine of the mice. The whole head of the animals containing the tumor tissues and the surrounding bone tissues were removed, fixed in $4 \%$ paraformaldehyde for $12 \mathrm{~h}$, and decalcified using $10 \%$ EDTA for 3 weeks. Subsequently, the whole head of the animal models were processed and embedded into paraffin wax using routine histological methods and cut into 5- $\mu$ m-thick sections. Finally, the sections were stained with H\&E. The sections were stained with Carrazi's hematoxylin (MUTO Pure Chemical Co., Ltd.) for $5 \mathrm{~min}$ at room temperature and stained with eosin (MUTO Pure Chemical Co., Ltd.) for $7 \mathrm{~min}$ at room temperature. The tumor part in the tissue of the animals was photographed under a bright field microscope (x4, x10, x20 and x40 magnification). The effects of VSCC-SCs, SCC-SCs and HDFs on the differentiation of OSCC cells in vivo were determined by the keratinized area and the size of the tumor nest according to the pathological diagnosis. The keratinized area indicated well-differentiated tissue and tumor nest formation represented moderate and poor differentiation. A small-sized tumor nest indicated poor differentiation and a medium-sized tumor nest indicated moderate differentiation.

Tartrate-resistant acid phosphatase (TRAP) staining. TRAP staining was conducted using a TRAP staining kit (Primary Cell, Co., Ltd.) according to the manufacturer's instructions. Active multi-nucleated osteoclast cells on the bone resorption 
surface were considered to be TRAP-positive cells. The interaction area contained tumor and bone in the tissue of whole animal model heads was photographed under a bright field microscope (x4, x10, x20 and x40 magnification). A total of five images (x40 magnification) were randomly acquired to obtain positive cell counts using ImageJ software (V1.51j8). Independent experiments were repeated three times.

Immunohistochemical analysis. Following antigen retrieval, the $5-\mu$ m-thick sections were blocked with $10 \%$ normal serum for $20 \mathrm{~min}$ at room temperature and then incubated with primary antibodies, including mouse anti-Ki67 (M7240, MIB1, 1:50; Dako; Agilent Technologies, Inc.), MMP9 (F-69, 56-2A4, 1:20; Fuji Yakukin Co., Ltd.) and MT1-MMP (F-86, 114-6G6, 1:20; Fuji Yakukin Co., Ltd.), overnight at $4^{\circ} \mathrm{C}$. After washing three times with TBS, all sections were incubated with the secondary antibody avidin-biotin complexes [PK-6102, mouse ABC kit; Blocking serum (normal serum, diluted with TBS): 1:75; biotinylated secondary antibody (diluted with normal serum): 1:200; reagent A (Avidin, ABC Elite) and reagent B (biotinylated HRP, ABC Elite, diluted by TBS): 1:55; Vector Laboratories, Inc.] for $1 \mathrm{~h}$ at room temperature. Following visualization with a mixed solution of $\mathrm{DAB} / \mathrm{H}_{2} \mathrm{O}_{2}$ (histofine DAB substrate; Nichirei Biosciences Inc.), The tumor areas containing the tumor and stroma in the tissue of the heads of the animals were photographed under a bright field microscope (x4, x10, x20 and x40 magnification). A total of five images (x40 magnification) were randomly acquired to obtain positive cell counts using ImageJ software (V1.51j8). The percentage of positive Ki-67/MMP9/MT1-MMP cells was calculated as the number of positive Ki-67/MMP9/MT1-MMP cancer cells/the number of all cancer cells x $100 \%$. Independent experiments were repeated three times.

Double-fluorescent immunohistochemical staining. Following antigen retrieval, the sections were blocked with Block Ace (DS Pharma Biomedical Co., Ltd.) for $20 \mathrm{~min}$ at room temperature and then incubated with primary antibodies, rabbit anti-Snail + SLUG (ab180714, 1:200; Abcam), and mouse anti-E-cadherin (M126, clone SHE78-7, 1:1,000, Takara Bio, Inc.), overnight at $4^{\circ} \mathrm{C}$. After washing three times with TBS, the sections were incubated with the secondary antibodies, anti-mouse IgG Alexa Fluor 488 (A21441, 1:100; Life Technologies; Thermo Fisher Scientific, Inc.) and anti-rabbit IgG Alexa Fluor 568 (A10042, 1:100; Life Technologies; Thermo Fisher Scientific, Inc.) for $1 \mathrm{~h}$ without light at room temperature. Finally, the sections were stained with $0.2 \mathrm{~g} / \mathrm{ml}$ DAPI (Dojindo Molecular Technologies, Inc.). The tumor areas containing the tumor and stroma in the tissue of the whole heads of the animals were photographed under a fluorescence microscope (x5, x10 and x20 magnification). The percentage of positive Snail cells was calculated as the number of positive snail cancer cells/the number of all cancer cells $x 100 \%$. Independent experiments were repeated three times.

Microarray and bioinformatics analyses. The VSCC-SCs, SCC-SCs and HDFs were used to conduct the microarray analysis. The HDFs were used as the control group to analyze the differentially expressed genes (DEGs) in the VSCC-SCs and SCC-SCs. The $\mid \operatorname{LogFCl}>1$ was considered as the cut-off value (https://www.ncbi.nlm.nih.gov/geo/query/acc. cgi?\&acc=GSE164374 (the dataset is currently private and is scheduled to be released on January 1, 2024). Biological processes and cell pathways of the upregulated DEGs were analyzed using Gene Ontology (GO) and Kyoto Encyclopedia of Genes and Genomes (KEGG) enrichment analysis through Cytoscape 3.7.2 (https://cytoscape. org/), and the results are presented as bubble plots created using $\mathrm{R}$ 3.6.2. An adjusted $\mathrm{P}<0.05$ was considered as the cut-off value. The top 10 hub genes were analyzed using a protein-to-protein interaction network (PPI) using STRING (http://string-db.org/) and Cytoscape 3.7 .2 (cytohubba). A combined score $>0.4$, was considered as the cut-off value, and the hub genes were selected according to the degree. Finally, the hub genes that were differentially expressed in the VSCC-SCs, SCC-SCs and HDFs were identified using cluster and heatmap analyses.

Statistical analysis. All statistical analyses were conducted using GraphPad Prism 9 (GraphPad Software, Inc.). Data are presented as the mean \pm standard deviation (SD). Two-way ANOVA was used to compare two variables with Tukey's post hoc test. One-way ANOVA was used to compared differences between $>2$ groups. The post hoc test used following one-way ANOVA was Tukey's. $\mathrm{P}<0.05$ was considered to indicate a statistically significant difference.

\section{Results}

VSCC-SCs, SCC-SCs and HDFs have a spindle-shaped morphology and suitable cell viability without cancer cell contamination. The morphology of the VSCC-SCs, SCC-SCs and HDFs was confirmed by Giemsa staining and IF. The spindle shape of the cells in the SCC-SCs group was the most notable, closely followed by those in the VSCC-SCs group. The spindle-shaped morphology of the cells in VSCC-SCs group was more evident than that of the cells in the HDFs group (Fig. 1A and B). In addition, IF staining revealed that the VSCC-SCs, SCC-SCs and HDFs were vimentin-positive and $\mathrm{AE} 1 / 3$-negative, which indicated that these three types of cells did not contain cancer cells (Fig. 1B). Finally, the viability of the VSCC-SCs, SCC-SCs and HDFs was examine using MTS assay. The results revealed that the OD values of the VSCC-SCs, SCC-SCs and HDFs were very similar on days 1 and 3, whereas they differed on day 5 . The OD value of the VSCC-SCs was the highest on day 5, followed by those of the HDFs group. The OD value of the cells in the HDFs group was slightly higher than that of the cells in the SCC-SCs group on day 5 (Fig. 1C). These data thus indicated that the VSCC-SCs and SCC-SCs exhibited a more obvious spindle-shaped morphology and suitable cell viability without cancer cell contamination.

Both VSCC-SCs and SCC-SCs suppress the tumor nest formation of $\mathrm{HSC}-3$ cells in vitro. Giemsa and IF staining were used to evaluate the effects of VSCC-SCs, SCC-SCs and HDFs on the tumor nest formation of HSC-3 cells in vitro. In the HSC-3 cell group, the cancer cells presented cellular pleomorphism; thus, the HSC-3 cells had strong cytological atypia. 
A

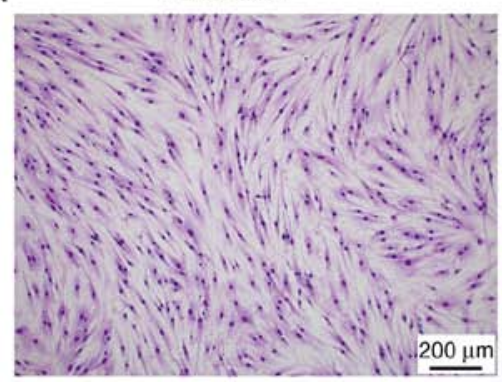

B
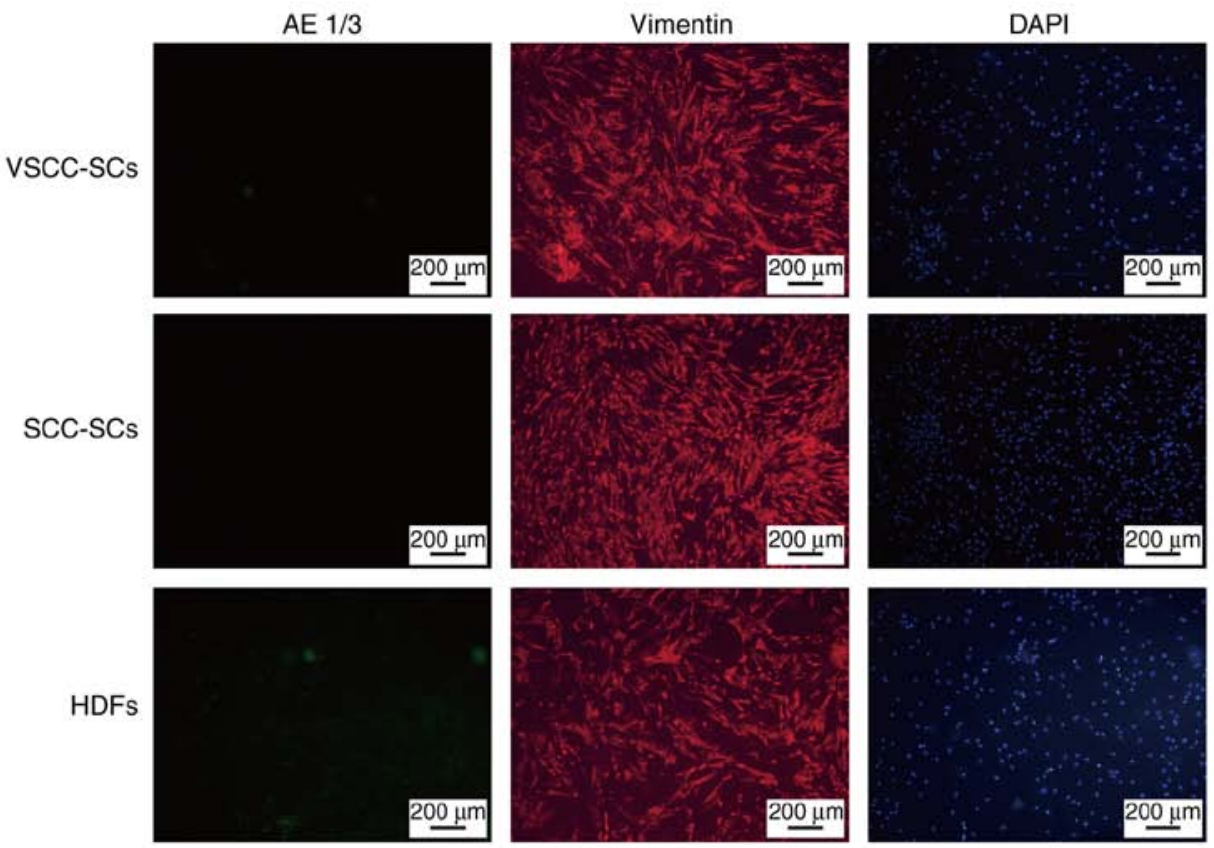

$200 \mu \mathrm{m}$
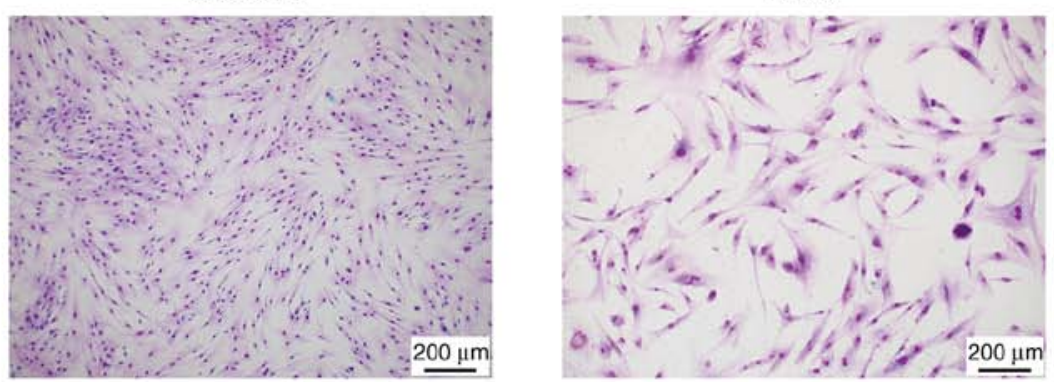

HDFs
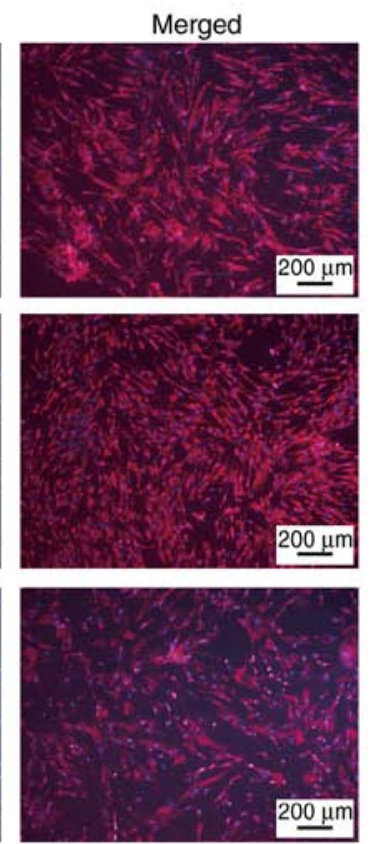

C

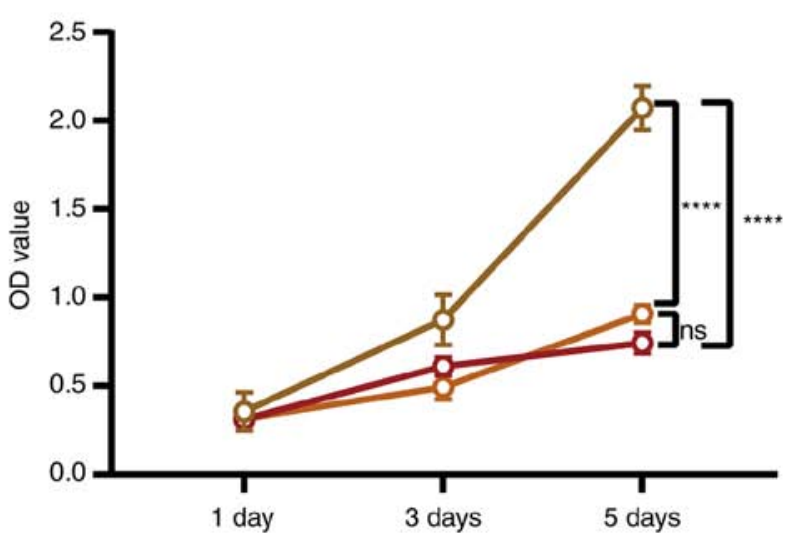

Figure 1. Examination of morphology and viability of VSCC-SCs, SCC-SCs and HDFs. (A and B) Morphology and cell component of VSCC-SCs, SCC-SCs and HDFs were tested by (A) Giemsa staining and (B) immunofluorescence staining. (C) Viability of VSCC-SCs, SCC-SCs and HDFs was examined by MTS assay on days 1, 3 and 5. Data are presented as the mean $\pm \mathrm{SD}, \mathrm{n}=4$. Statistical analysis was performed using two-way ANOVA; ns, not significant (P>0.05) and ${ }^{* * * *} \mathrm{P}<0.0001$. VSCC-SCs, verrucous squamous cell carcinoma-associated stromal cells; SCC-SCs, squamous cell carcinoma-associated stromal cells; HDFs, human dermal fibroblasts.

In the HSC-3 + SCC-SCs group, the adhesion of cancer cells was weak, there was no obvious cancer nest formation, and the cancer cells infiltrated diffusely into the stroma (Fig. 2A and $\mathrm{B})$. Furthermore, the average number of cells in the tumor nests in the HSC-3 + SCC-SCs group was the lowest, followed by the HSC-3 + VSCC-SCs group. In addition, there was a minimal difference between the HSC-3 and HSC-3 + HDFs groups (Fig. 2C). Therefore, both the VSCC-SCs and SCC-SCs inhibited the tumor nest formation of HSC-3 in vitro, and the SCC-SCs exerted a more potent inhibitory effect than the VSCC-SCs, while the HDFs exerted a minimal effect on the tumor nest formation of HSC-3 cells in vitro.

VSCC-SCs and SCC-SCs promote the proliferation of HSC-3 cells in vitro. The effects on cell proliferation between VSCC-SCs and HSC-3 cells were examined using MTS assay. 
A

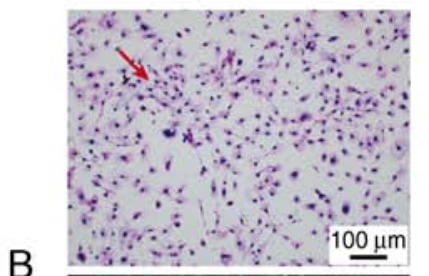

B
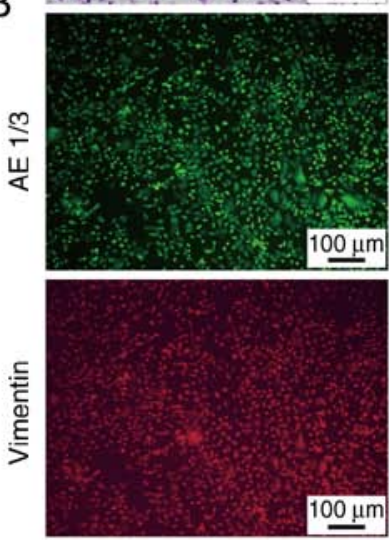

$\overline{\frac{\pi}{\Delta}}$
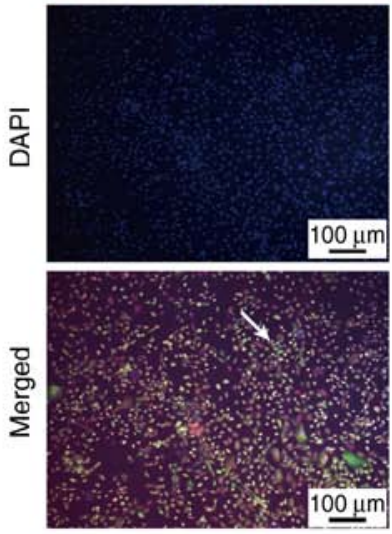

HSC-3+VSCC-SCs
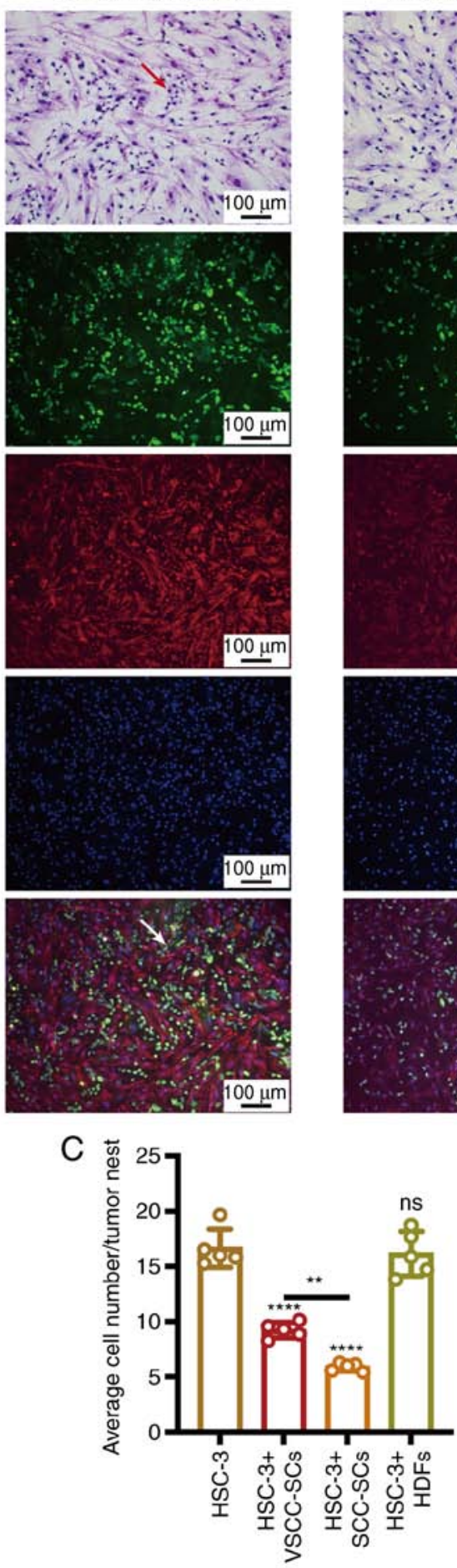

HSC-3+SCC-SCs
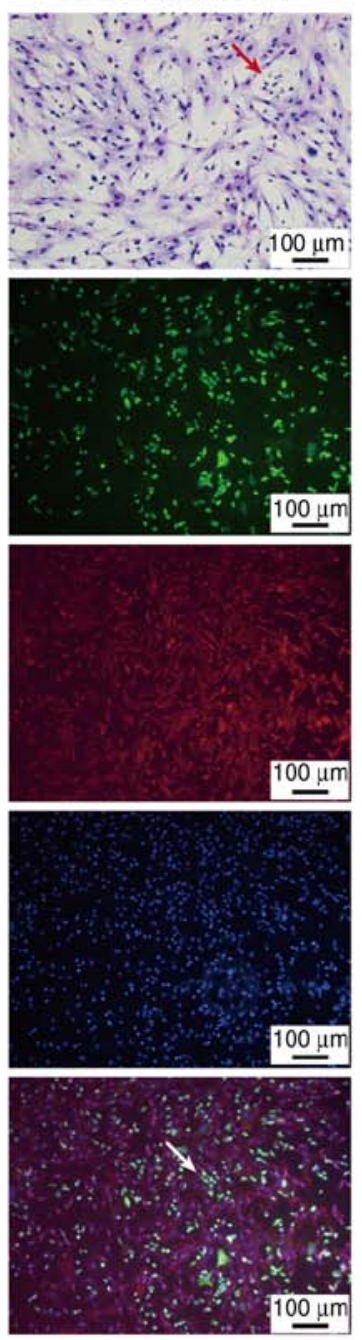

HSC-3+HDFs
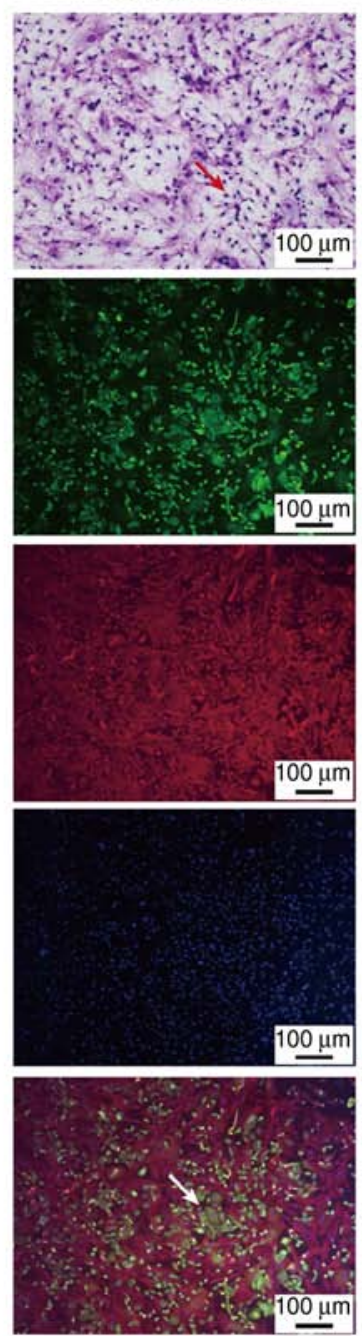

Figure 2. Both VSCC-SCs and SCC-SCs suppress the tumor nest formation of HSC-3 in vitro. (A and B) Effect of VSCC-SCs, SCC-SCs and HDFs on the tumor nest formation of HSC-3 in vitro was examined using both (A) Giemsa staining and (B) immunofluorescence staining. The arrows in the images indicate the tumor nest. (C) Quantification of average cells number of tumor nest in the different groups. Data are presented as the mean $\pm S D, n=5$. Statistical analysis was performed using one-way ANOVA; ns, not significant $(\mathrm{P}>0.05) ;{ }^{* *} \mathrm{P}<0.01$ and ${ }^{* * * * *} \mathrm{P}<0.0001$, all compared with the HSC-3 group. VSCC-SCs, verrucous squamous cell carcinoma-associated stromal cells; SCC-SCs, squamous cell carcinoma-associated stromal cells; HDFs, human dermal fibroblasts.

The results demonstrated that there was a promoting effect on cell proliferation in the HSC-3 + VSCC-SCs on day 3 , while proliferation was inhibited on day 5 compared with the VSCC-SCs (Fig. 3A). The effect on the proliferation between the SCC-SCs and HSC-3 cells was also examined by MTS assay. The results demonstrated that there was promoting effect observed in the HSC-3 + SCC-SCs on days 1 and 5 (Fig. 3B).
In addition, the effect on cell proliferation between HDFs and HSC-3 was examined by MTS assay, which demonstrated that there was a promoting effect observed in the HSC-3 + HDFs on days 1 and 3 (Fig. 3C). In addition, the OD value of the HSC-3 (2,000 cells), HSC-3 + VSCC-SCs and HSC-3 + HDFs groups markedly increased on day 3 and decreased slightly on day 5, while that of the HSC-3 + SCC-SCs group increased 
A

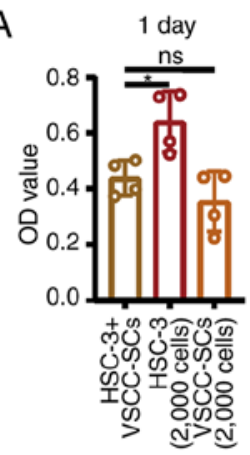

C

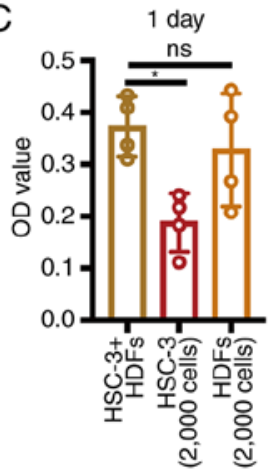

E

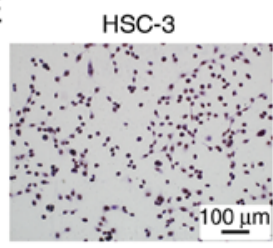

3 days
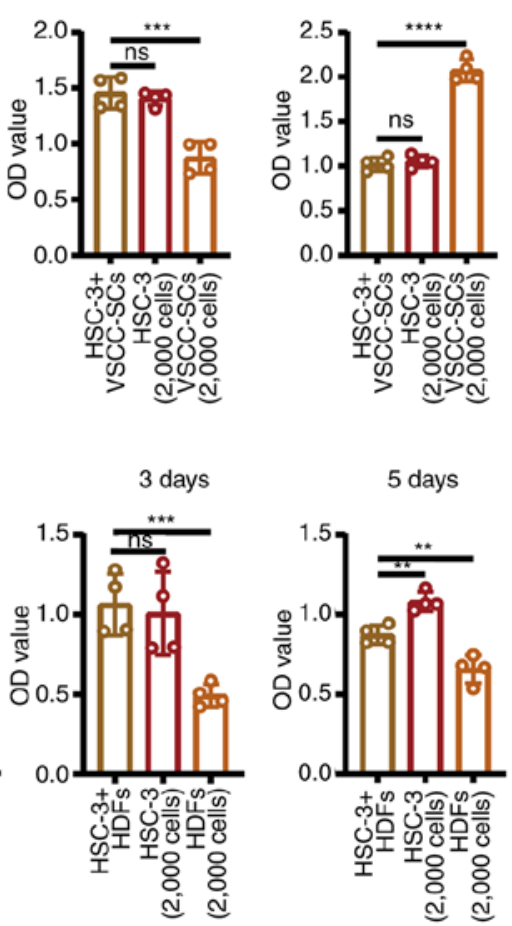

D

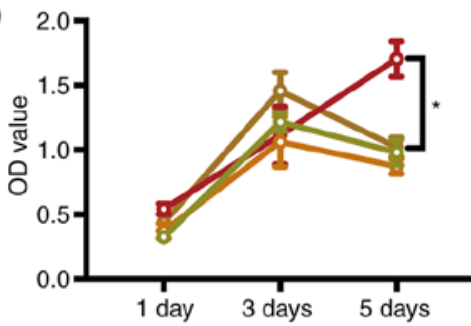

B
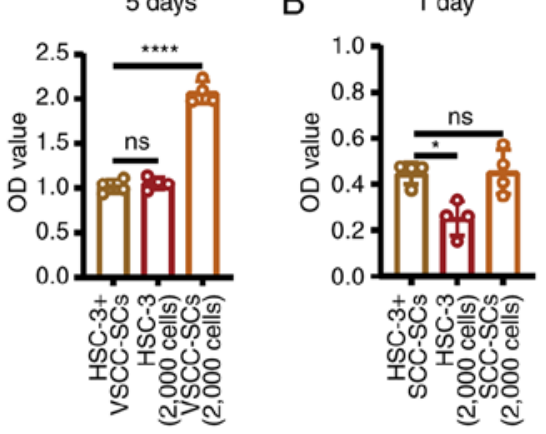
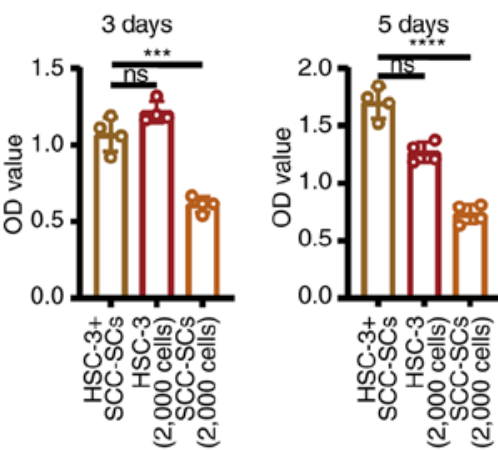

-HSC-3+VSCC-SCs

-HSC-3+SCC-SCs

-HSC-3+HDFs

$\multimap$ HSC-3 (2,000 cells)

Figure 3. Both VSCC-SCs and SCC-SCs promote the proliferation of HSC-3 cells in vitro. (A-C) MTS assay was used to examine the effect on the proliferation between VSCC-SCs, SCC-SCs, HDFs and HSC-3 at 1, 3 and 5 days. Data are presented as the mean \pm SD, n=4. Statistical analysis was performed using one-way ANOVA; ns, not significant $(\mathrm{P}>0.05) ;{ }^{*} \mathrm{P}<0.05,{ }^{* * *} \mathrm{P}<0.01,{ }^{* * * *} \mathrm{P}<0.001,{ }^{* * * *} \mathrm{P}<0.0001$. (D) Comparison of the effect on the proliferation between VSCC-SCs, SCC-SCs, HDFs and HSC-3 on days 1, 3 and 5. Data are presented as the mean \pm SD, $n=4$. Statistical analysis was performed using two-way ANOVA; ${ }^{\mathrm{P}}<0.05$. (E) Immunohistochemistry was used to examine the relative Ki-67 expression level to assay the effect of VSCC-SCs, SCC-SCs and HDFs on the proliferation of HSC-3 in vitro. (F) Quantification of positive Ki-67 cell numbers in different groups; data are presented as the mean \pm SD, $n=3$. Statistical analysis was performed using one-way ANOVA; ns, not significant $(\mathrm{P}>0.05) ;{ }^{* * *} \mathrm{P}<0.001$, compared with the HSC-3 group. VSCC-SCs, verrucous squamous cell carcinoma-associated stromal cells; SCC-SCs, squamous cell carcinoma-associated stromal cells; HDFs, human dermal fibroblasts.

gradually from days 1 to 5 (Fig. 3D). These data suggested that there was a promoting effect on cell proliferation between the VSCC-SCs, SCC-SCs, HDFs and HSC-3 cells in vitro. Furthermore, the relative expression of $\mathrm{Ki}-67$ in the different groups was examined by IHC to determine the effects of the VSCC-SCs, SCC-SCs and HDFs on the proliferation of HSC-3 cells in vitro (Fig. 3E). The percentage of positive Ki-67 cells in the HSC-3 + SCC-SCs group was slightly higher than that in the HSC-3 + VSCC-SCs group, which was significantly higher than that in the HSC-3 + HDFs and HSC-3 groups (Fig. 3F). These data suggested that both the VSCC-SCs and SCC-SCs promoted the proliferation of the HSC-3 cells in vitro and that the SCC-SCs exert a more prominent promoting effect than the VSCC-SCs.

Both the VSCC-SCs and SCC-SCs promote the invasion of $H S C-3$ cells in vitro. A Transwell (invasion) assay and IF staining were used to determine the effects of VSCC-SCs, SCC-SCs and HDFs on the invasion of HSC-3 cells in vitro (Fig. 4A). The number of invading cancer cells in the HSC-3 + SCC-SCs group was slightly higher than that in the HSC-3 + VSCC-SCs group and markedly higher than that in the HSC-3 + HDFs and HSC-3 $(10,000$ cells) groups. Furthermore, the number of invasive cancer cells in the HSC-3 + HDFs group was slightly lower than that in the HSC-3 (10,000 cells) group (Fig. 4B). These data indicated that both the VSCC-SCs and SCC-SCs promoted the invasion of HSC-3 cells in vitro, while the HDFs exerted an inhibitory effect on the invasion of HSC-3 cells. In addition, SCC-SCs exerted a more prominent promoting effect than the VSCC-SCs.

Both the VSCC-SCs and SCC-SCs promote the migration of HSC-3 cells in vitro. A Transwell (migration) assay and IF staining were used to determine the effects of VSCC-SCs, SCC-SCs and HDFs on the migration of HSC-3 cells 
A

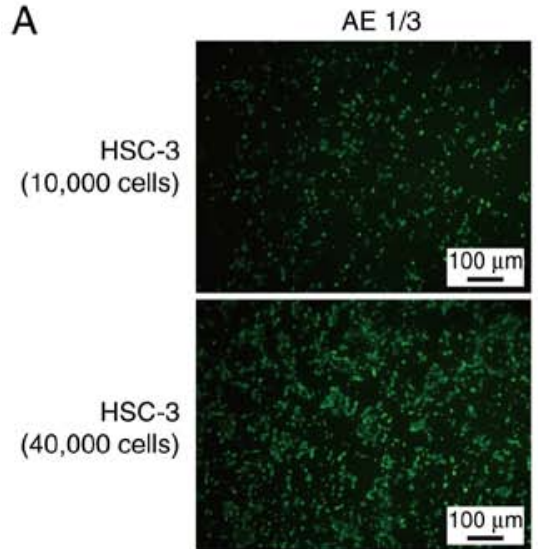

AE 1/3
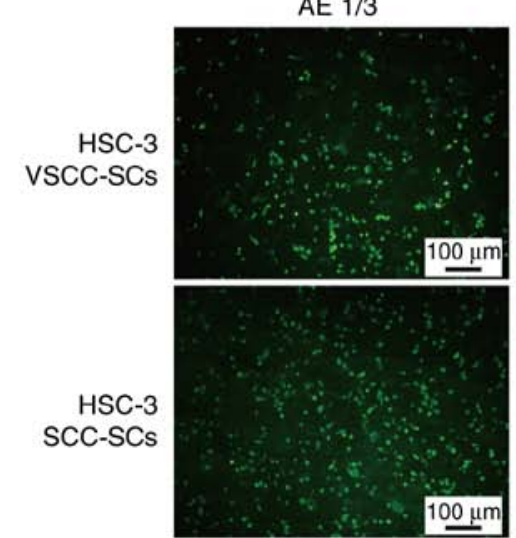

$100 \mu \mathrm{m}$

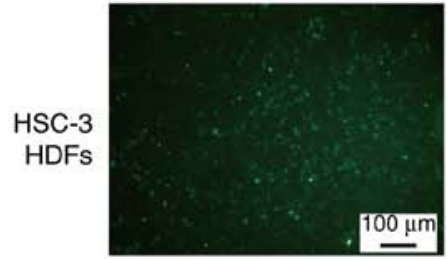

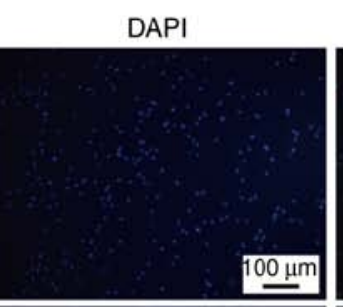

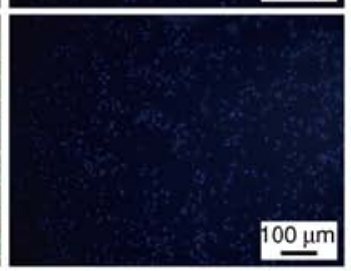

Vimentin
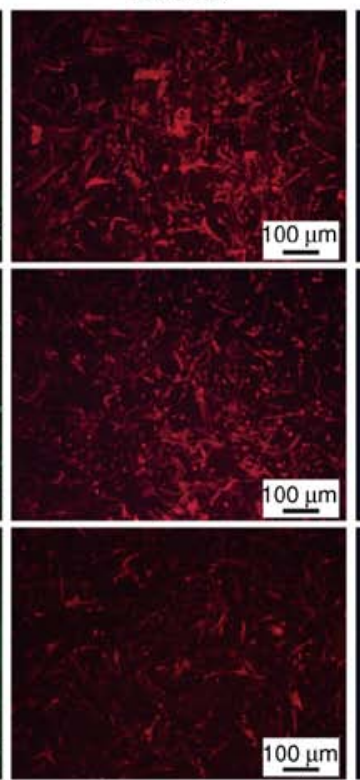

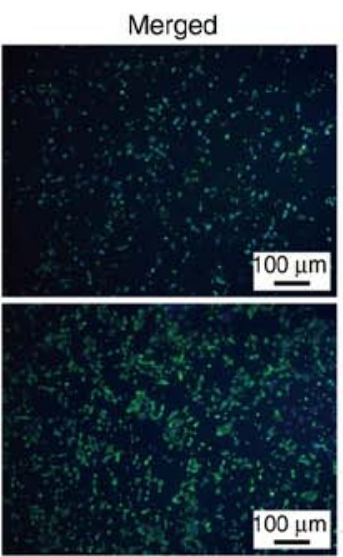

DAPI
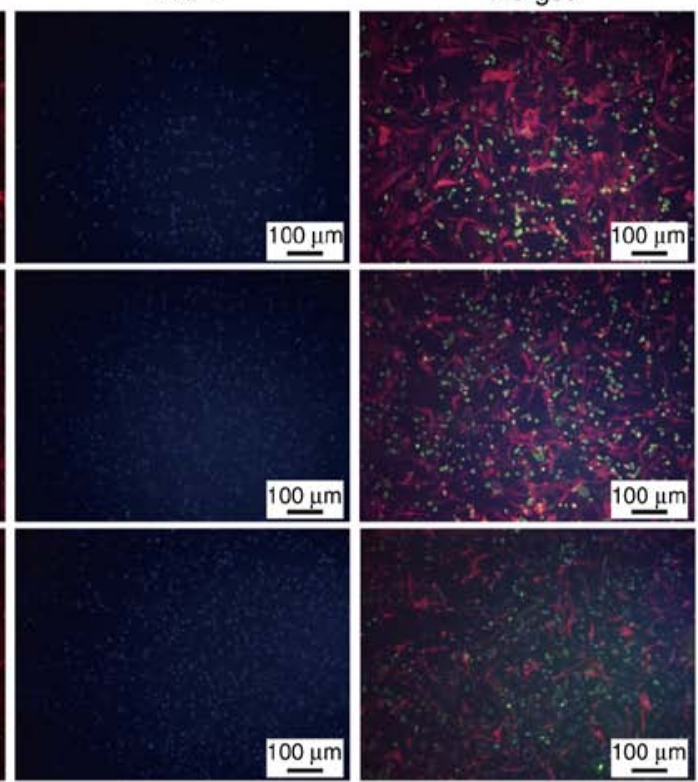
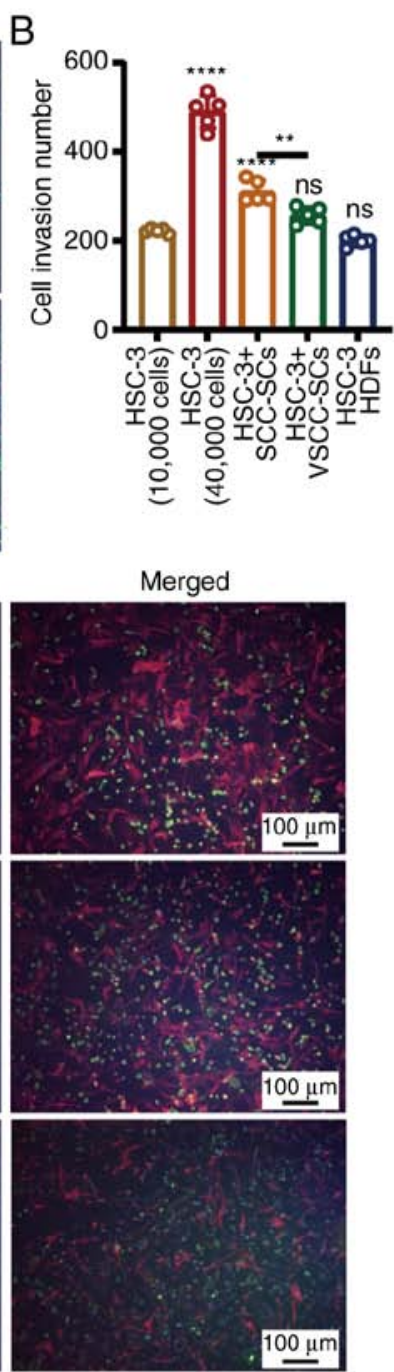

Figure 4. Both VSCC-SCs and SCC-SCs enhance the invasive ability of HSC-3 cells in vitro. (A) Both Transwell (invasion) and immunofluorescence staining assays were used to examine the effects of VSCC-SCs, SCC-SCs and HDFs on the invasion of HSC-3 cells. Green color indicates HSC-3 cells and red color indicates stromal cells ( $\mathrm{x} 5$ magnification). (B) Quantification of cell invasion number in different groups. Data are presented as the mean $\pm \mathrm{SD}, \mathrm{n}=5$. Statistical analysis was performed using one-way ANOVA; ns, not significant $(\mathrm{P}>0.05) ;{ }^{* *} \mathrm{P}<0.01$ and ${ }^{* * * *} \mathrm{P}<0.0001$, all compared with the HSC-3 (10,000 cells) group. VSCC-SCs, verrucous squamous cell carcinoma-associated stromal cells; SCC-SCs, squamous cell carcinoma-associated stromal cells; HDFs, human dermal fibroblasts.

in vitro (Fig. 5A). The number of migrating cells in the HSC-3 + SCC-SCs group was slightly higher than that in the HSC-3 + VSCC-SCs group, and markedly higher than that in the HSC-3 + HDFs and HSC-3 (5,000 cells) groups. Furthermore, the number of migrating cells in the HSC-3 + HDFs group was slightly lower than that in the HSC-3 (5,000 cells) group (Fig. 5B). These data thus indicated that both the VSCC-SCs and SCC-SCs promoted the migration of HSC-3 cells in vitro, while the HDFs exerted an inhibitory effect on the migration of HSC-3 cells. In addition, the SCC-SCs exerted a more prominent promotion effect than the VSCC-SCs.

$S C C$-SCs inhibit the differentiation of OSCC in vivo, whereas VSCC-SCs promote the differentiation of OSCC cells. H\&E staining was used to evaluate the effects of VSCC-SCs, SCC-SCs and HDFs on the differentiation of OSCC cells in vivo. In the HSC-3 only group, tumor tissue formation with a small cancer nest without keratinization was observed, and histological analysis revealed moderately differentiated OSCC. In the HSC-3 + SCC-SCs group, H\&E staining revealed that the cancer nests were slightly smaller than those in the HSC-3 only group. Histological analysis of the HSC-3 + SCC-SCs group revealed moderately differentiated type OSCC. In the HSC-3 + VSCC-SCs and HSC-3 + HDFs groups, these histological findings differed markedly compared with the HSC-3 only group. In the HSC-3 + VSCC-SCs and HSC-3 + HDFs groups, well-differentiated OSCC with notable keratinization in the center of the tumor tissue was observed (Fig. 6). Thus, these data indicated that the SCC-SCs inhibit the differentiation of OSCC in vivo, while the VSCC-SCs and HDFs promoted the differentiation of OSCC.

Both the VSCC-SCs and SCC-SCs promote OSCC proliferation invivo. The expression of $\mathrm{Ki}-67$ in different groups was assessed by IHC to demonstrate the effect of VSCC-SCs, SCC-SCs and HDFs on the proliferation of OSCC cells in vivo (Fig. 7A). Compared with the HSC-3 only group, the expression of Ki-67 was slightly increased in the HSC-3 + VSCC-SCs group and was markedly increased in the HSC-3 + SCC-SCs group. In addition, a minimal effect was observed in the HSC-3 + HDFs 
A

HSC-3

(5,000 cells)
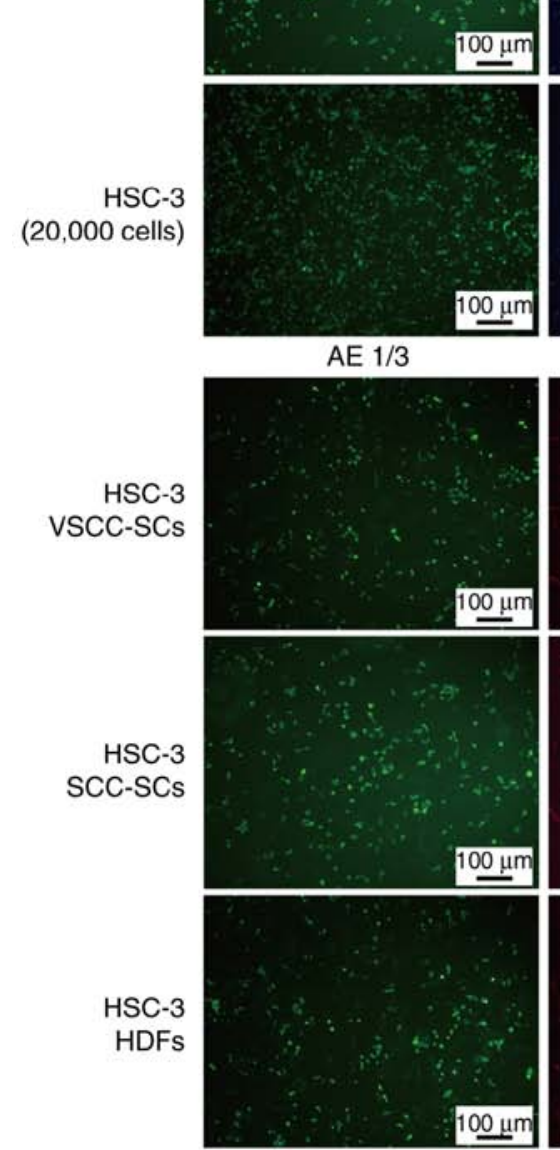

$\mathrm{AE} 1 / 3$

AE 1/3
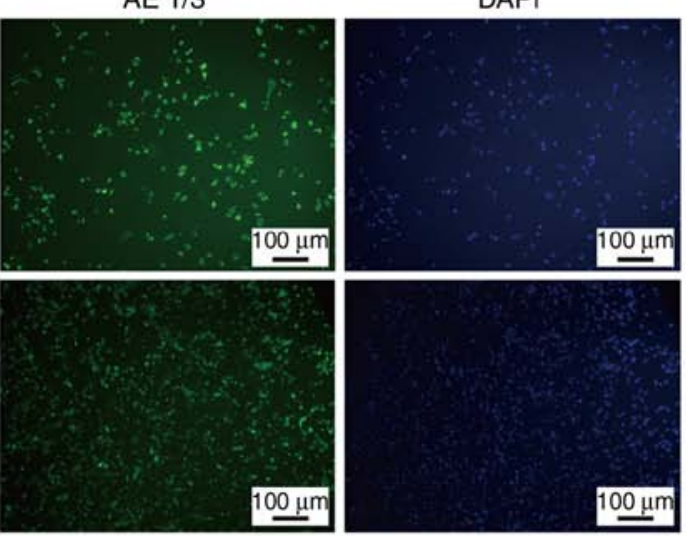

Vimentin
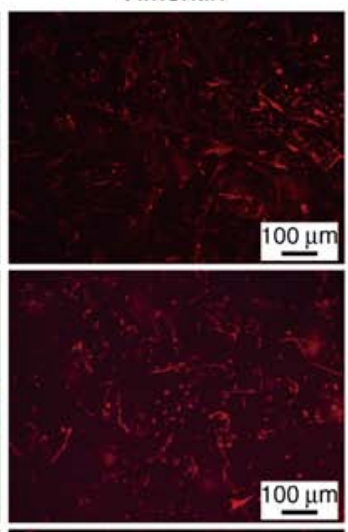

DAPI
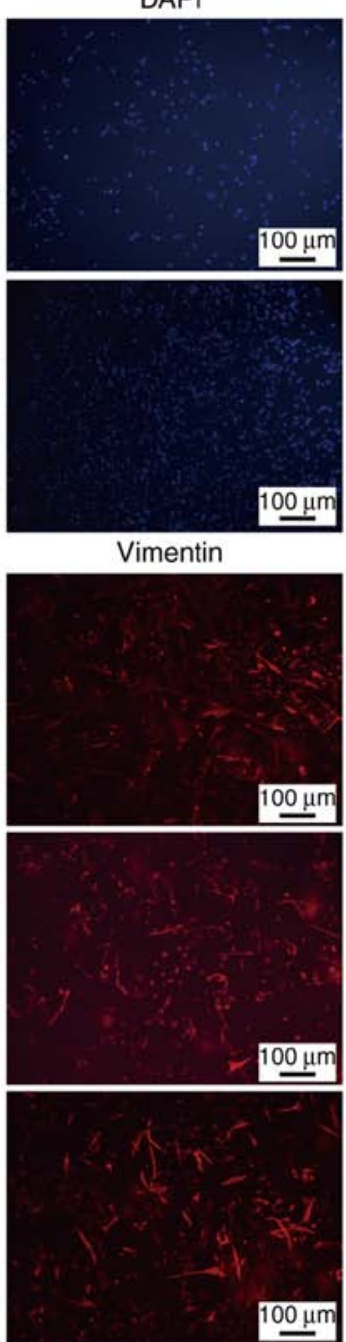

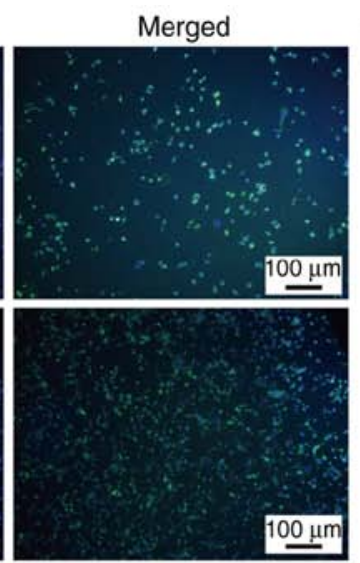

DAPI
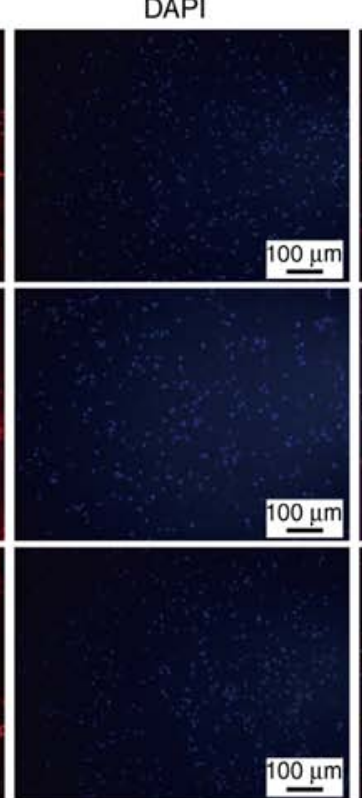

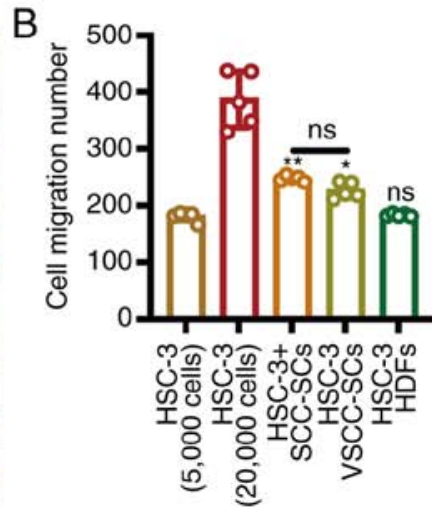

Merged

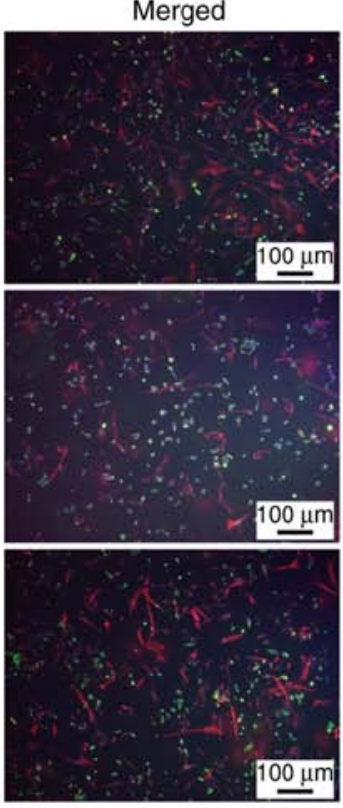

Figure 5. Both VSCC-SCs and SCC-SCs enhance the migratory ability of HSC-3 cells in vitro. (A) Transwell (migration) and immunofluorescence staining assays were used to examine the effects of VSCC-SCs, SCC-SCs and HDFs on the migration of HSC-3 cells. Green color indicates HSC-3 cells and red color indicates stromal cells ( $\mathrm{x} 5$ magnification). (B) Quantification of cell migration number in different groups. Data are presented as the mean $\pm \mathrm{SD}$, $\mathrm{n}=5$. Statistical analysis was performed using one-way ANOVA; ns, not significant ( $\mathrm{P}>0.05)$; ${ }^{*} \mathrm{P}<0.05$ and ${ }^{* *} \mathrm{P}<0.01$, all compared with the HSC-3 (5,000 cells) group. VSCC-SCs, verrucous squamous cell carcinoma-associated stromal cells; SCC-SCs, squamous cell carcinoma-associated stromal cells; HDFs, human dermal fibroblasts.

group (Fig. 7B). Thus, these data suggested that both the VSCC-SCs and SCC-SCs promoted the proliferation of OSCC in vivo and that SCC-SCs exert a more prominent promoting effect than the VSCC-SCs.

Both the VSCC-SCs and SCC-SCs promote the bone invasion and invasion of OSCC cells in vivo. The effect of VSCC-SCs, SCC-SCs and HDFs on the bone resorption of OSCC were evaluated by H\&E staining. Bone resorption in the HSC-3 + SCC-SCs group was slightly greater than that in the VSCC-SCs group, while it was markedly greater than that in the HSC-3 + HDFs group. There was a minimal difference between the HSC-3 and HSC-3 + HDFs groups (Fig. 8A). Furthermore, TRAP staining was used to determine the number of active multinucleated osteoclasts in the different groups to demonstrate the effects of VSCC-SCs, SCC-SCs and HDFs on the bone invasion of OSCC (Fig. 8B). The number of active multinucleated osteoclasts in the HSC-3 + SCC-SCs group was the highest, followed by the HSC-3+VSCC-SCs and HSC-3 groups. In addition, the number of active multinucleated osteoclasts in the HSC-3 + HDFs groups was slightly lower than that in the HSC-3 group (Fig. 8C). These data suggest that the VSCC-SCs and SCC-SCs promoted the bone invasion of OSCC in vivo and that SCC-SCs exert a more prominent promoting effect, while the HDFs exerted an inhibitory effect. The expression levels of MMP9 and MT1-MMP, markers of invasion, in the different groups were assessed by IHC to determine the effects of VSCC-SCs, SCC-SCs and HDFs on the invasion of OSCC cells in vivo (Fig. 8D and F). Compared with the HSC-3 group, the number of cells positive for MMP9 and MT1-MMP in the HSC-3 + VSCC-SCs and HSC-3 + SCC-SCs groups markedly increased, while there was a minimal effect in the HSC-3 + HDFs group (Fig. 8E and G). Therefore, both the VSCC-SCs and SCC-SCs promoted the invasion of OSCC cells in vivo, while the HDFs exerted a minimal effect. These data suggest that both the VSCC-SCs and SCC-SCs promote the bone invasion, and the invasion of OSCC in vivo.

Both VSCC-SCs and SCC-SCs promote the EMT of OSCC cells in vivo. The EMT biomarkers, E-cadherin and Snail, were examined by IF staining to demonstrate the effects of VSCC-SCs, 

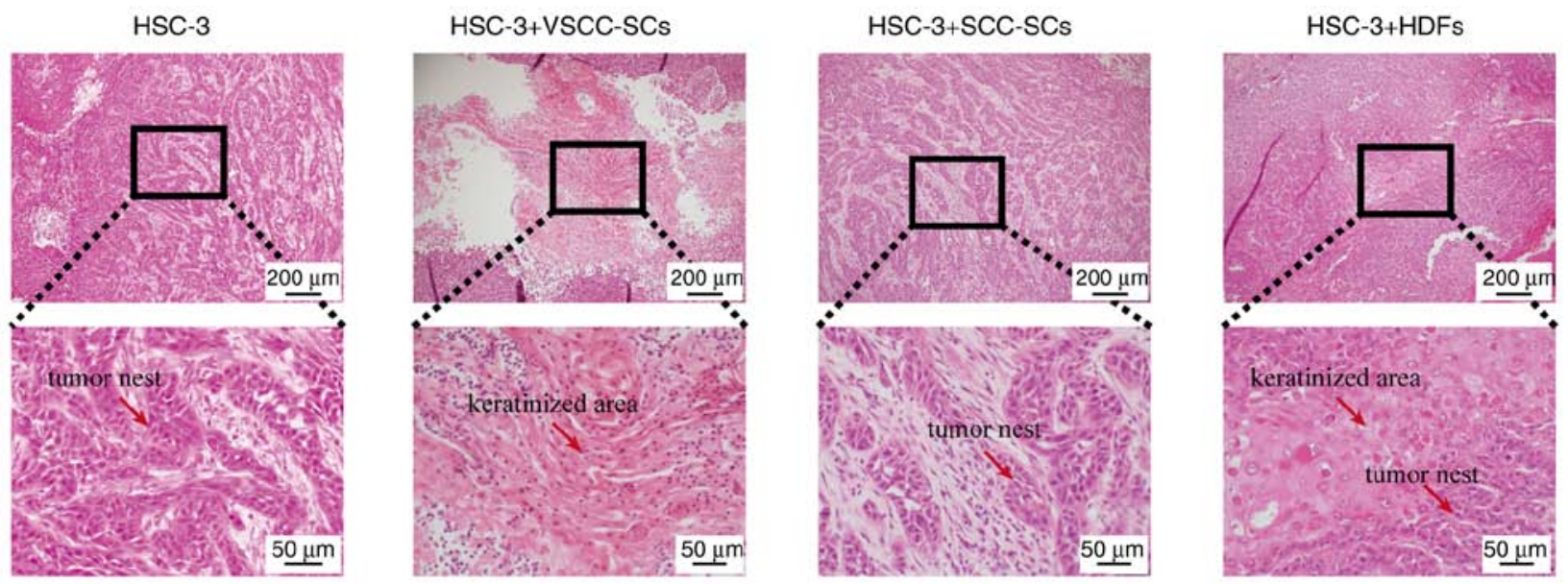

Figure 6. SCC-SCs inhibit the differentiation of OSCC, while VSCC-SCs promote the differentiation of OSCC in vivo. H\&E staining was used to examined the effects of VSCC-SCs, SCC-SCs and HDFs on the differentiation of OSCC in vivo. VSCC-SCs, verrucous squamous cell carcinoma-associated stromal cells; SCC-SCs, squamous cell carcinoma-associated stromal cells; HDFs, human dermal fibroblasts; OSCC, oral squamous cell carcinoma.

A

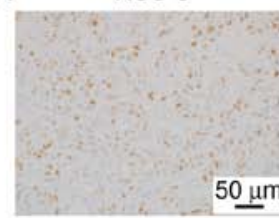

HSC-3+VSCC-SCS

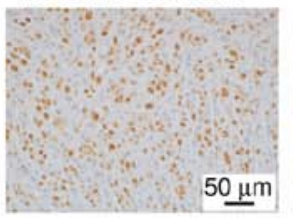

HSC-3+SCC-SCs

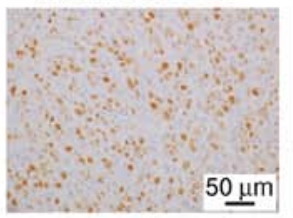

HSC-3+HDFS

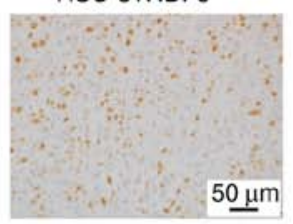

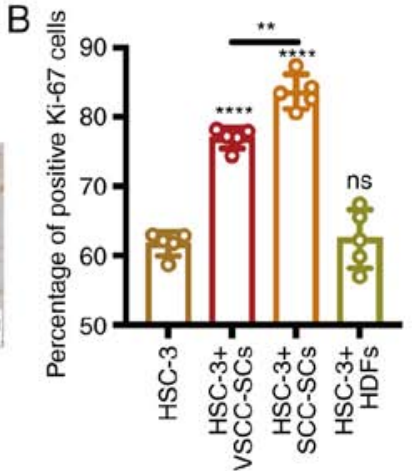

Figure 7. Both VSCC-SCs and SCC-SCs promote the proliferation of OSCC in vivo. (A) Immunohistochemical staining was used to examine the relative expression of Ki-67 to determine the effect of VSCC-SCs, SCC-SCs and HDFs on the proliferation of OSCC cells in vivo. (B) Quantification of positive Ki-67 cells in the different groups. Data are presented as the mean $\pm \mathrm{SD}, \mathrm{n}=5$. Statistical analysis was performed using one-way ANOVA; ns, not significant (P>0.05); ${ }^{* *} \mathrm{P}<0.01$ and $^{* * * *} \mathrm{P}<0.0001$ compared with the HSC-3 group. VSCC-SCs, verrucous squamous cell carcinoma-associated stromal cells; SCC-SCs, squamous cell carcinoma-associated stromal cells; HDFs, human dermal fibroblasts; OSCC, oral squamous cell carcinoma.

SCC-SCs and HDFs on the EMT process of OSCC (Fig. 9A). The percentage of positive Snail cells in the HSC-3 + SCC-SCs group was slightly higher than that in the HSC-3 + VSCC-SCs groups and markedly higher than that in the HSC-3 and HSC-3 + HDFs groups. In addition, the percentage of positive Snail cells in HSC-3 + HDFs group was lower than that in the HSC-3 group (Fig. 9B). Thus, these data suggested that both the VSCC-SCs and SCC-SCs promoted the EMT process of OSCC and that the SCC-SCs exerted a more prominent promoting effect than the VSCC-SCs, while the HDFs exerted an inhibitory effect on the EMT process of OSCC in vivo.

$C$-X-C motif chemokine ligand (CXCL)8, $C$ - $C$ motif chemokine ligand 2 (CCL2), $C-X-C$ motif chemokine ligand 1 (CXCL1), mitogen-activated protein kinase 3 (MAPK3) and phosphatidylinositol-4,5-bisphosphate 3-kinase catalytic subunit alpha (PIK3CA) may be involved in the regulation of OSCC progression by VSCC-SCs and SCC-SCs. DEGs in the VSCC-SCs and SCC-SCs were analyzed using a microarray and compared with those in the HDFs. Common upregulated DEGs between the VSCC-SCs and SCC-SCs were identified using a Venn diagram, which indicated that 1,123 genes were upregulated in both the VSCC-SCs and SCC-SCs (Fig. 10A). Furthermore, the cancer-associated biological process and cancer-associated cell pathways of the common upregulated DEGs were analyzed using GO and KEGG enrichment analysis, respectively (Fig. 10B and C). Common upregulated DEGs were mainly enriched in cancer-associated biological processes, such as cell proliferation, cell adhesion and cell migration (Fig. 10B). In addition, common upregulated-DEGs were mainly enriched in cancer-associated cellular pathways, such as the TNF signaling pathway, pathways in cancer, IL-17 signaling pathway and cytokine-cytokine receptor interaction (Fig. 10C). Common upregulated DEGs both associated with cancer biological processes and cancer cell pathways were identified using the Venn diagram; 76 common upregulated DEGs were both associated with cancer biological processes and cancer cell pathways (Fig. 10D). The top 10 hub genes in these common upregulated DEGs both associated with cancer biological processes and cancer cell pathways were analyzed using a PPI network. CXCL8, MAPK3, prostaglandin-endoperoxide synthase 2 (PTGS2), bone morphogenetic protein 4 (BMP4), CD40, hepatocyte growth factor (HGF), EGFR, PIK3CA, CXCL1 and CCL2 were identified as the hub genes 

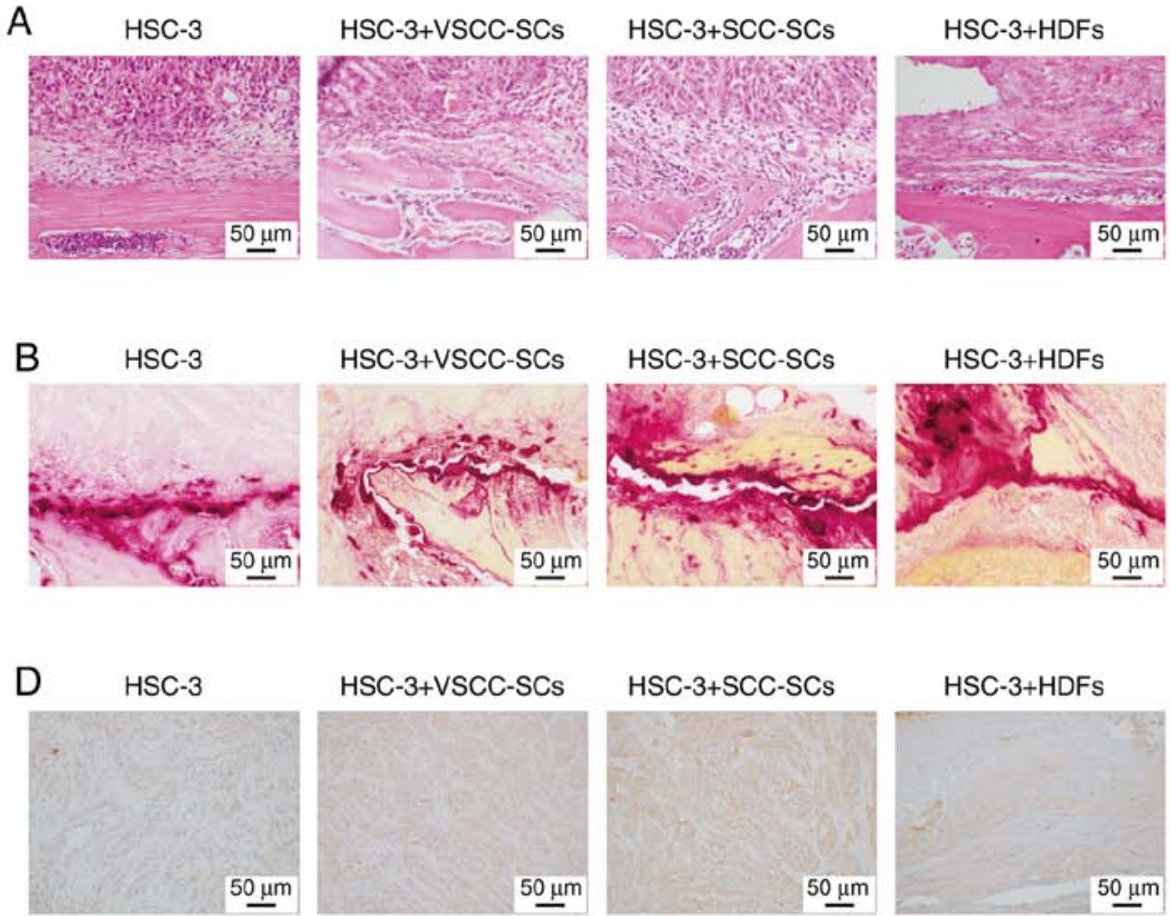

HSC-3+VSCC-SCs
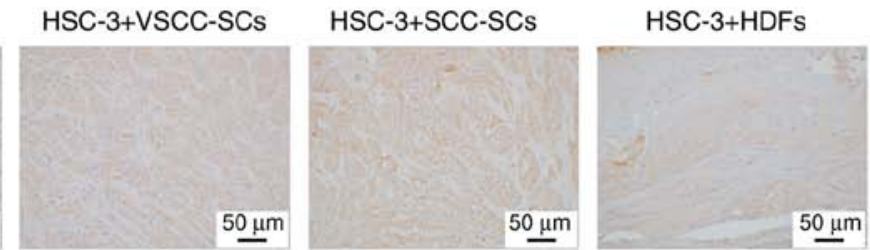

F HSC-3
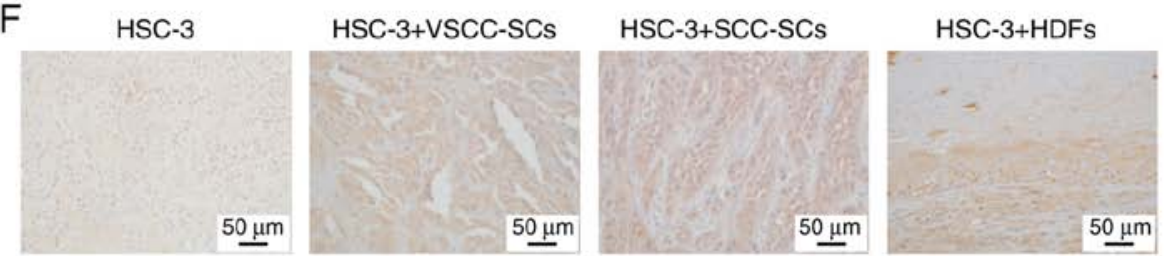
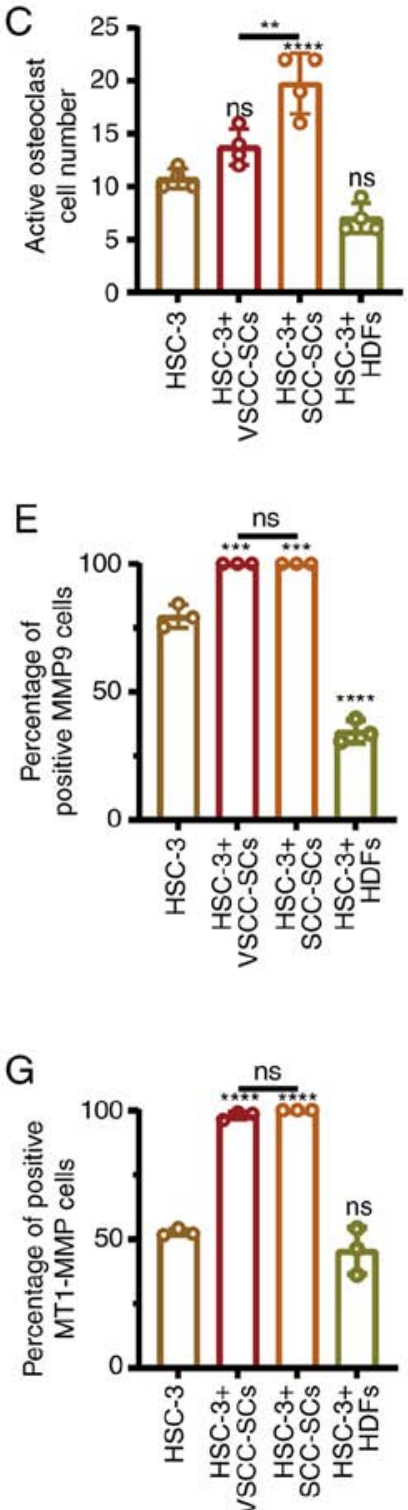

Figure 8. Both VSCC-SCs and SCC-SCs promote the bone invasion and invasion of OSCC in vivo. (A and B) Effect of VSCC-SCs, SCC-SCs and HDFs on the bone invasive ability of OSCC in vivo was examine by H\&E and TRAP staining. (C) Quantification of activated multi-nucleated osteoclast cell number in the different groups. Data are presented as the mean $\pm \mathrm{SD}, \mathrm{n}=3$. Statistical analysis was performed using one-way ANOVA; ns, not significant (P $>0.05$ ); ${ }^{* *} \mathrm{P}<0.01$ and ${ }^{* * * *} \mathrm{P}<0.0001$, all compared with the HSC-3 group. (D and F) MMP9 and MT1-MMP expression levels in the different groups were examined by immunohistochemistry. (E and G) Quantification of positive MMP9 and MT1-MMP cells in the different groups. Data are presented as the mean \pm SD, $\mathrm{n}=5$. Statistical analysis was performed using one-way ANOVA; ns, not significant $(\mathrm{P}>0.05)$; ${ }^{* * *} \mathrm{P}<0.001$ and ${ }^{* * * * *} \mathrm{P}<0.0001$, all compared with the HSC-3 group. VSCC-SCs, verrucous squamous cell carcinoma-associated stromal cells; SCC-SCs, squamous cell carcinoma-associated stromal cells; HDFs, human dermal fibroblasts; OSCC, oral squamous cell carcinoma; TRAP, tartrate-resistant acid phosphatase.

(Fig. 10E). Finally, the top 10 hub genes differentially expressed in VSCC-SCs, SCC-SCs, and HDFs were analyzed by cluster and heatmap analysis. CXCL8, MAPK3, PIK3CA, CXCL1 and CCL2 were differentially expressed in VSCC-SCs, SCC-SCs, and HDFs (Fig. 10F). These data suggest that CXCL8, CCL2, CXCL1, MAPK3 and PIK3CA may be involved in the regulation of OSCC progression by VSCC-SCs and SCC-SCs.

Intercellularadhesion molecule 1 (ICAM1), interleukin (IL)1B, Fos proto-oncogene, AP-1 transcription factor subunit (FOS), bone morphogenetic protein 4 (BMP4), insulin (INS) and nerve growth factor (NGF) may underlie the differential effects of VSCC-SCs and SCC-SCs on the differentiation of OSCC. The upregulated DEGs in the SCC-SCs were analyzed using a microarray and compared with those in the
VSCC-SCs. The biological processes of upregulated DEGs were analyzed using GO enrichment analysis. The upregulated DEGs were mainly enriched in cell differentiation, cell proliferation, cell adhesion and cell migration (Fig. 11A). Furthermore, the top 10 hub genes involved in cell differentiation were identified using a PPI network, as follows: IL6, ICAM1, IL1B, Cadherin 1 (CDH1), CDC42, FOS, Toll-like receptor (TLR)4, BMP4, INS and NGF (Fig. 11B). Finally, the hub genes differentially expressed in SCC-SCs and VSCC-SCs were analyzed using cluster and heatmap analyses, which suggested that ICAM1, IL1B, FOS, BMP4, INS and NGF were differentially expressed in the SCC-SCs (Fig. 11C). Therefore, ICAM1, IL1B, FOS, BMP4, INS and NGF may underlie the differential effects of VSCC-SCs and SCC-SCs on the differentiation of OSCC. 
A
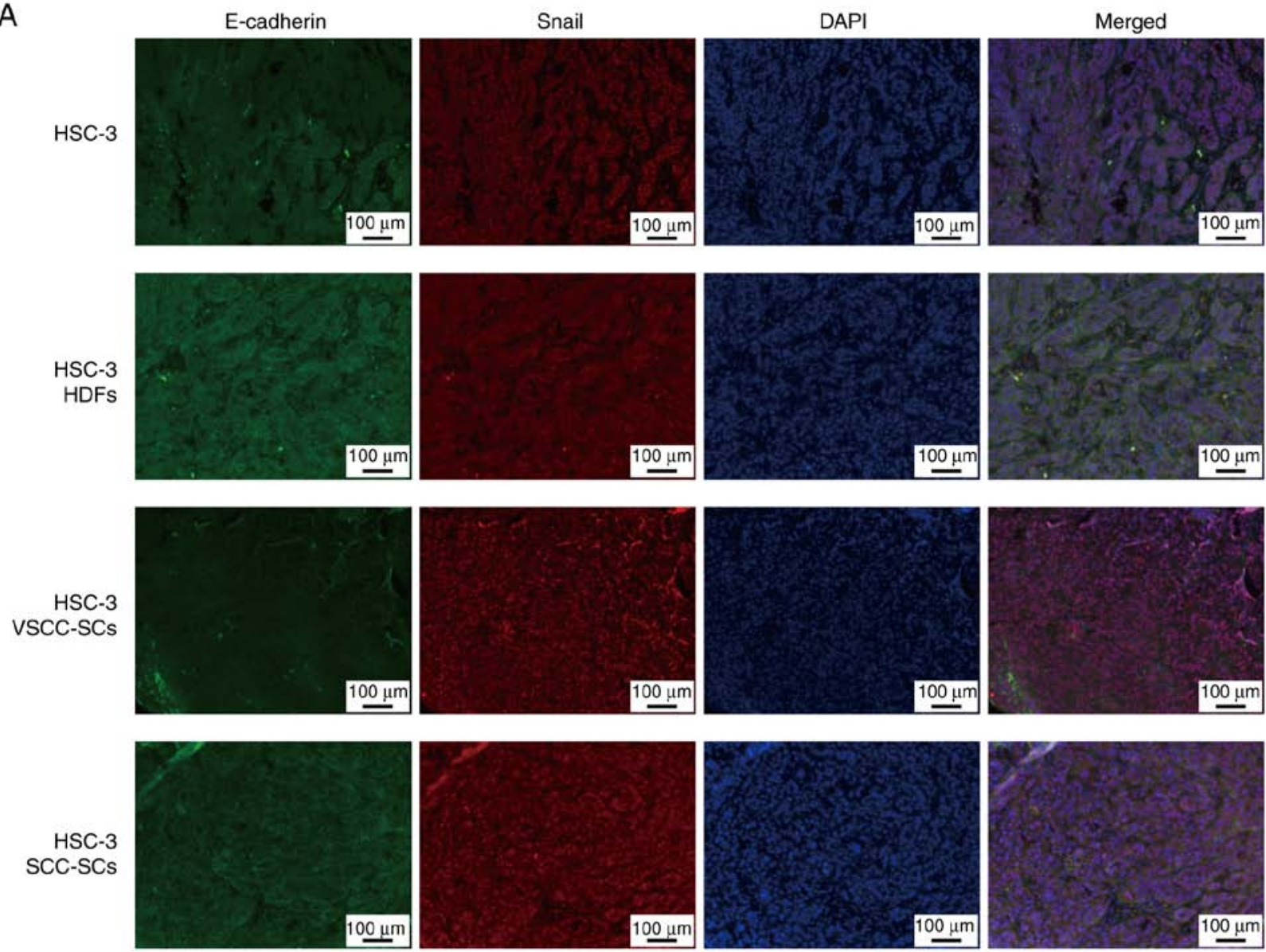

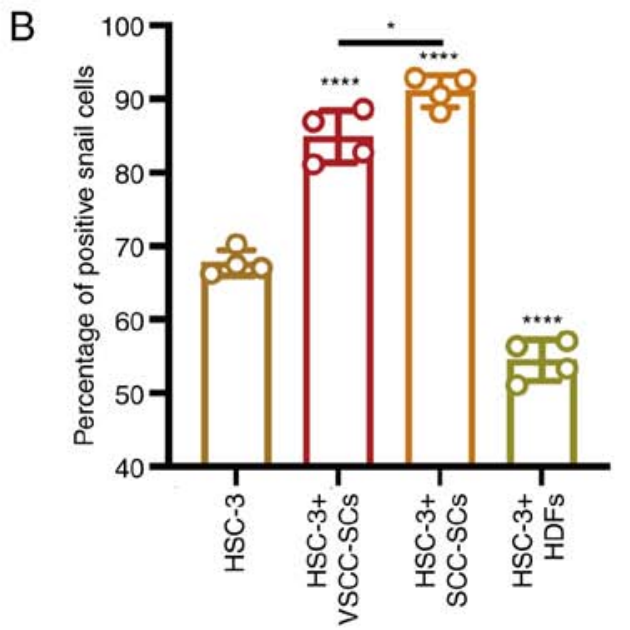

Figure 9. Both VSCC-SCs and SCC-SCs promote the EMT process of OSCC. (A) Effect of VSCC-SCs, SCC-SCs and HDFs on the EMT process of OSCC in vivo was examined by double-fluorescent immunohistochemical staining. (B) Quantification of positive snail cells in different groups. Data are presented as the mean $\pm \mathrm{SD}, \mathrm{n}=4$. Statistical analysis was performed using one-way ANOVA; ${ }^{*} \mathrm{P}<0.05$ and ${ }^{* * * *} \mathrm{P}<0.0001$, compared with the HSC-3 group. VSCC-SCs,verrucous squamous cell carcinoma-associated stromal cells; SCC-SCs, squamous cell carcinoma-associated stromal cells; HDFs, human dermal fibroblasts; OSCC, oral squamous cell carcinoma; EMT, epithelial-to-mesenchymal transition.

\section{Discussion}

Benign and malignant cancers can be assayed by the degree of differentiation. The prognosis of patients with well-differentiated cancers is better than that for patients with moderately differentiated and poorly differentiated cancers. Therefore, the degree of differentiation can influence the biological processes of cancers (20). It has been suggested that SRY-box transcription factor 4 (SOX4), SUMO-specific peptidase 3 (SENP3), grainyhead like transcription factor 2 (GRHL2) and RUNX family transcription factor 1 (RUNX1) are significantly associated with the differentiation of OSCC (21-24). In addition, the extracellular signal-regulated kinase $1 / 2$ (ERK1/2), JNK and STAT3 signaling pathways also play significant roles in the differentiation of OSCC (25). Moreover, S100A16 has been shown to promote the differentiation of OSCC cells and 
A

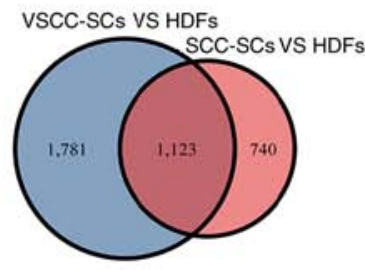

C

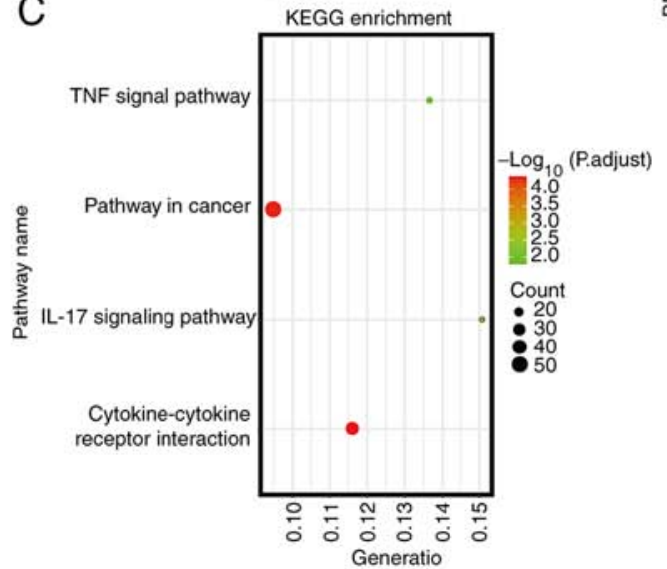

B
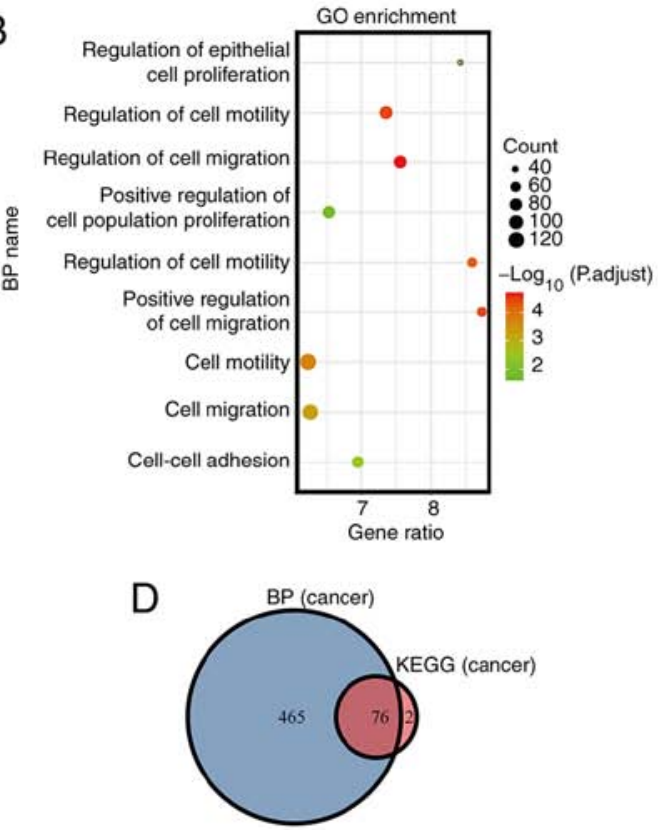

E
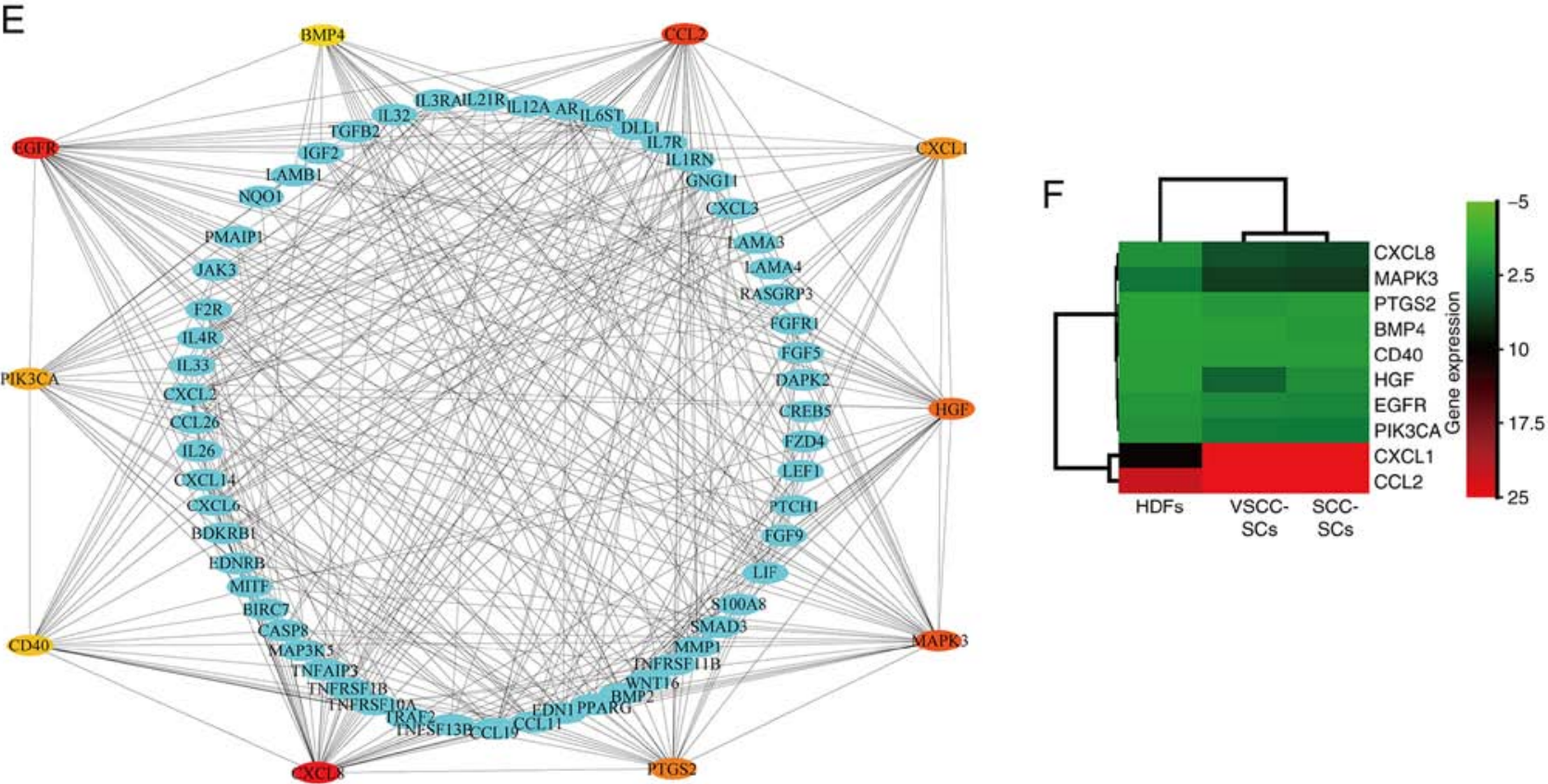

Figure 10. Potential genes in both VSCC-SCs and SCC-SCs that regulate the progression of OSCC examined using microarray analysis. (A) Common upregulated DEGs in both VSCC-SCs and SCC-SCs identified using a Venn diagram. (B and C) Cancer associated biological process and cancer associated cell pathway that common upregulated DEGs enriched in were examined using GO and KEGG enrichment analysis, respectively (only the biological process and cell pathways associated with the progression of cancer are presented). (D) Common upregulated DEGs both associated with cancer biological process and cancer cell pathways were identified by Venn. (E) PPI network was used to identify the top 10 hub genes in common upregulated DEGs associated with cancer biological process and cancer cell pathways. (F) Top 10 hub genes differentially expressed in VSCC-SCs, SCC-SCs and HDFs were identified using cluster analysis and a heatmap. VSCC-SCs, verrucous squamous cell carcinoma-associated stromal cells; SCC-SCs, squamous cell carcinoma-associated stromal cells; HDFs, human dermal fibroblasts; OSCC, oral squamous cell carcinoma; GO, Gene Ontology; KEGG, Kyoto Encyclopedia of Genes and Genomes; PPI network, protein-to-protein interaction network.

inhibit the progression of OSCC (26). Therefore, the differentiation of OSCC may be regulated by specific proteins and signaling pathways, which can further influence its progression. However, to the best of our knowledge, no study to date has investigated the effects of stromal cells on the differentiation of OSCC. In the present study, it was found that VSCC-SCs promoted the differentiation of OSCC, while SCC-SCs inhibited the differentiation of OSCC. HDFs exerted a minimal effect on the differentiation of OSCC cells. Furthermore, the reason for the differential effects of VSCC-SCs and SCC-SCs on the degree of OSCC differentiation was analyzed using microarray and bioinformatics methods. The results indicated that the overexpression of ICAM1, IL1B, FOS, BMP4, INS and NGF in the SCC-SCs was closely associated with cell differentiation. It has been suggested that ICAM1 is closely associated with the differentiation of osteoclasts and can also regulate the differentiation of colorectal cancer (27-29); however, it has not been widely investigated in the differentiation of OSCC. 

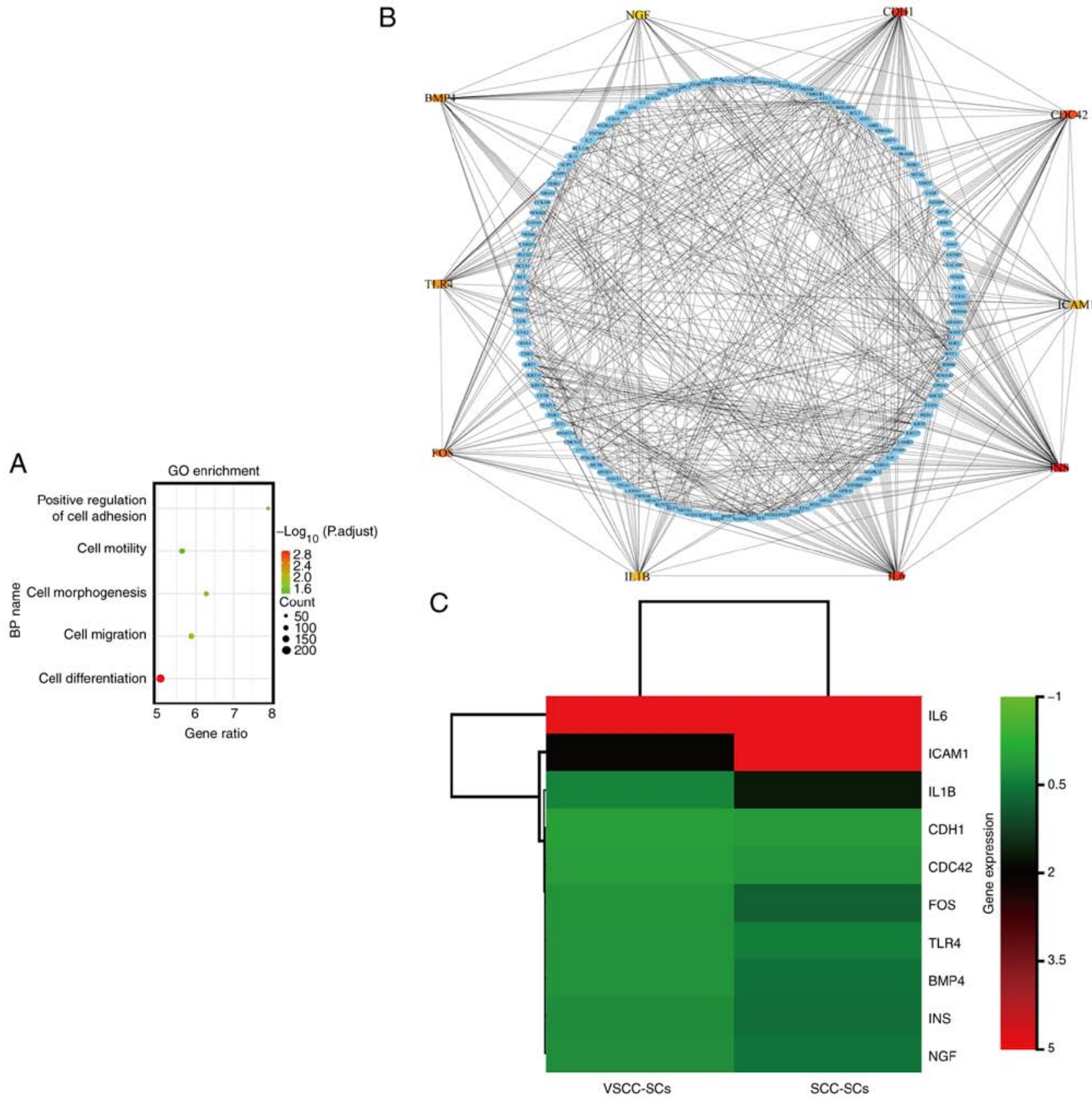

Figure 11. Identification of potential genes resulting in the differential effects of VSCC-SCs and SCC-SCs on the differentiation of OSCC. (A) Biological process of upregulated DEGs in SCC-SCs examined using GO enrichment analysis. (B) Top 10 hub genes in cell differentiation were analyzed using a PPI network and Cytoscape software. (C) The hub genes differentially expressed in SCC-SCs and VSCC-SCs were identified using cluster analysis and a heatmap. VSCC-SCs, verrucous squamous cell carcinoma-associated stromal cells; SCC-SCs, squamous cell carcinoma-associated stromal cells; HDFs, human dermal fibroblasts; OSCC, oral squamous cell carcinoma; GO, Gene Ontology; PPI network, protein-to-protein interaction network.

IL1B regulates the differentiation of TH17 and TH19 in the TME $(30,31)$. IL1B also plays a critical role in the differentiation of prostate cancer cells (32). BMP4 plays a significant role in the differentiation of neuroblastoma, colorectal cancer stem cells and $\mathrm{CD}_{133^{+}}$hepatic cancer stem cells; however, it has not been extensively studied in the differentiation of OSCC (33-35). NGF regulates the differentiation of neuroblasts and neuroblastoma cells $(36,37)$. FOS (also known as p55) and INS have not been extensively investigated in the differentiation of OSCC. Therefore, ICAM1, IL1B, BMP4 and NGF may underlie the differential effects of VSCC-SCs and SCC-SCs on the degree of differentiation of OSCC. Stromal cells, which are the main components of the TME, are also associated with cancer progression $(7,38)$. A previous study suggested that the proliferation, invasion and metastasis of CD133+ pancreatic cancer cells was enhanced following co-culture with pancreatic stromal cells (39). Senescent stromal cells promote the growth and invasion of CRC both in vitro and in vivo (40). In addition, the results of a gene expression microarray in three types of cancer demonstrated that stromal cells could alter cellular functions, including cell-cycle, cell signal, cell movement and cell death (41). The results of these studies indicated that stromal cells influence the progression of multiple cancer types. As regards OSCC, a recent study suggested that CAFs-derived exosomal miR-382-5p promoted the invasion and migration of OSCC (42). Long 
non-coding RNAs (lncRNAs) also play a significant role in CAFs, promoting the progression of OSCC (43). Epiregulin has been found to promote the transformation of normal fibroblasts into CAFs, which are induced in OSCC via the JAK2/STAT3 pathway (44). Therefore, miRNAs and IncRNAs in CAFs promote the progression of OSCC. However, research investigating the crosstalk between stromal cells and OSCC is limited (17). The findings of the present study indicated that VSCC-SCs and SCC-SCs promoted the proliferation, invasion and migration of OSCC both in vitro and in vivo, and that SCC-SCs exerted a more prominent promoting effect than the VSCC-SCs, while HDFs exert a limited effect on the proliferation, invasion and migration of OSCC. In addition, the proliferation of HSC-3 + VSCC-SCs and HSC-3 + HDFs decreased on day 5, whereas that of HSC-3 + SCC-SCs increased on day 5 in vitro, according to the results of MTS assay; this may be caused by differences in the viability of VSCC-SCs, SCC-SCs and HDFs. Cell death in the HSC-3 + VSCC-SCs and HSC-3 + HDFs was observed from day 5 due to the higher viability of VSCC-SCs and HDFs, which resulted in cell crowding. Conversely, the death of HSC-3 + SCC-SCs was not observed due to the lower viability of SCC-SCs, which did not lead to cell crowding on day 5. Finally, SCC-SCs inhibited the degree of OSCC differentiation, indicating that SCC-SCs have the potential to promote the proliferation, invasion and migration of OSCC. The results obtained for proliferation, invasion and migration indirectly confirmed differentiation. Thus, SCC-SCs inhibited the differentiation, and promoted the proliferation, invasion, and migration of OSCC cells. VSCC-SCs exerted a promoting effect on the differentiation degree of OSCC, indicating that VSCC-SCs have the potential to inhibit the proliferation, invasion and migration of OSCC. However, apart from the keratinized area, an area of invasion was also observed in the HSC-3 + VSCC-SCs group based on H\&E staining. Thus, the effect of VSCC-SCs on the proliferation, invasion and migration of OSCC could not be identified simply by the degree of differentiation. The results indicated that the VSCC-SCs promoted the proliferation, invasion and migration of OSCC cells. Thus, VSCC-SCs promoted the differentiation, proliferation, invasion and migration of OSCC cells. Therefore, both the VSCC-SCs and SCC-SCs promote the progression of OSCC, while the HDFs exerted a minimal effect. In addition, the SCC-SCs exerted a more prominent promoting effect than the VSCC-SCs.

In addition, the microarray data in the present study also suggested that CXCL8, CCL2, CXCL1, MAPK3 and PIK3CA in the VSCC-SCs and SCC-SCs may regulate the progression of OSCC. CXCL8 (also known as IL-8) regulates cellular responses in combination with CXCR1 and CXCR2 (45). A recent study demonstrated that the loss of androgen receptor could promote the expression of CXCL8 in CAFs, which in turn regulates the migration of prostate cancer cells (46). In addition, blocking autophagy in CAFs can promote the progression of HNSCC by regulating the secretion of IL6, IL8, and the other cytokines (47). Therefore, CXCL8 has the potential to regulate the crosstalk between stromal cells and OSCC. CCL2, which is expressed in both cancer and stromal cells, recruits the macrophages during acute inflammation by binding to $\mathrm{CCR} 2$, a process that can regulate the immunoreactivity of the TME $(48,49)$. The results of a recent study indicated that CCL2-mediated inflammation increases the risk of breast cancer (50). CCL2 also mediates the crosstalk between breast cancer and stromal cells that regulate the breast cancer stem cells (51). Thus, CCL2 plays a significant role in the TME of multiple cancer types, and has the potential to regulate the crosstalk between stromal cells and OSCC. CXCL1, which is expressed in both cancer and stromal cells, regulates cancer progression by binding to CXCR2 $(52,53)$. A recent study indicated that CXCL1 in CAFs promoted the proliferation of ovarian cancer by the activation of p38 (54). TGF- $\beta$ and IL1B regulate the progression of breast cancer and OSCC by regulating CXCL1 in CAFs respectively $(55,56)$. Therefore, CXCL1 in stromal cells has the potential to regulate the progression of OSCC. MAPK3 (also known as ERK1) plays a significant role in the progression of multiple types of cancer $(57,58)$. The results of a recent study suggested that the crosstalk between cancers and stromal cells is suppressed upon ERK1/2 inhibition in cancer-associated pancreatic stellate cells (59). Therefore, MAPK3 also plays a significant role in the crosstalk between cancer and stromal cells, and has the potential to mediate the crosstalk between stromal cells and OSCC. PIK3CA (also known as PI3K) plays a significant role in the progression of OSCC $(60,61)$. However, to the best of our knowledge, no study to date has investigated the role and function of PIK3CA in the crosstalk between stromal cells and OSCC.

In conclusion, the present study demonstrated that VSCC-SCs promoted the differentiation, proliferation, invasion and metastasis of OSCC, while the SCC-SCs inhibited differentiation and promoted the proliferation, invasion and metastasis of OSCC. Compared with the VSCC-SCs, the SCC-SCs exerted an inhibitory effect on the differentiation and exerted a more potent promoting effect on the proliferation, invasion and migration of OSCC. Finally, CXCL8, CCL2, CXCL1, MAPK3 and PIK3CA in VSCC-SCs and SCC-SCs may regulate the progression of OSCC, and ICAM1, IL1B, FOS, BMP4, INS and NGF may underlie the differential effects of VSCC-SCs and SCC-SCs on the differentiation of OSCC. These findings may describe a potential regulatory mechanism in the progression of OSCC.

\section{Acknowledgements}

Not applicable.

\section{Funding}

The present study was supported by the Japan Society for the Promotion of Science (JSPS) Grants-in-Aid for Scientific Research (KAKENHI; grant nos. JP18K0978900, JP18K 1722400，JP20H0388812，JP19K1915901, JP21K1004303 and JP21K1708903).

\section{Availability of data and materials}

The datasets used and/or analyzed during the current study are available from the corresponding author on reasonable request. In addition, the dataset GSE164374 is currently private and is scheduled to be released on Jan 01, 2024. 


\section{Authors' contributions}

QS designed the outline of the study. QS, HO, KT, MWO, $\mathrm{HK}, \mathrm{KN}, \mathrm{SI}, \mathrm{AS}$ and HN conducted the experiments and data analysis. QS, KT and HO were involved in the preparation of manuscript. QS wrote the manuscript. KN, SI, AS and HN supervised the study and contributed to data interpretation and manuscript revision. KT and HN confirm the authenticity of all raw data. All authors have read and agreed to the published version of the manuscript.

\section{Ethics approval and consent to participate}

The present study was approved by the Ethics Committee of Okayama University (project identification code: 1703-042-001). Informed consent was obtained from all patients.

\section{Patient consent for publication}

Not applicable.

\section{Competing interests}

The authors declare that they have no competing interests.

\section{References}

1. Lindemann A, Takahashi H, Patel AA, Osman AA and Myers JN Targeting the DNA damage response in OSCC with TP53 mutations. J Dent Res 97: 635-644, 2018.

2. Jiang Y, Li T, Wu Y, Xu H, Xie C, Dong Y, Zhong L, Wang Z, Zhao H, Zhou Y, et al: GPR39 overexpression in OSCC promotes YAP-sustained malignant progression.J Dent Res 99: 949-958, 2020.

3. Chen Y, Shao Z, Jiang E, Zhou X, Wang L, Wang H, Luo X, Chen Q, Liu K and Shang Z: CCL21/CCR7 interaction promotes EMT and enhances the stemness of OSCC via a JAK2/STAT3 signaling pathway. J Cell Physiol 235: 5995-6009, 2020

4. Yang Z, Liang X, Fu Y, Liu Y, Zheng L, Liu F, Li T, Yin X, Qiao X and $\mathrm{Xu} \mathrm{X}$ : Identification of AUNIP as a candidate diagnostic and prognostic biomarker for oral squamous cell carcinoma. EBioMedicine 47: 44-57, 2019.

5. Quail DF and Joyce JA: Microenvironmental regulation of tumor progression and metastasis. Nat Med 19: 1423-1437, 2013.

6. Li Z, Liu FY and Kirkwood KL: The p38/MKP-1 signaling axis in oral cancer: Impact of tumor-associated macrophages. Oral Oncol 103: 104591, 2020.

7. Yoshihara K, Shahmoradgoli M, Martínez E, Vegesna R, Kim H, Torres-Garcia W, Treviño V, Shen H, Laird PW, Levine DA, et al: Inferring tumour purity and stromal and immune cell admixture from expression data. Nat Commun 4: 2612, 2013.

8. Peddareddigari VG, Wang D and Dubois RN: The tumor microenvironment in colorectal carcinogenesis. Cancer Microenviron 3 : 149-166, 2010.

9. Bhome R, Mellone M, Emo K, Thomas GJ, Sayan AE and Mirnezami AH: The colorectal cancer microenvironment: Strategies for studying the role of cancer-associated fibroblasts. Methods Mol Biol 1765: 87-98, 2018.

10. Udagawa $\mathrm{T}$ and Wood M: Tumor-stromal cell interactions and opportunities for therapeutic intervention. Curr Opin Pharmacol 10: 369-374, 2010.

11. Potter SM, Dwyer RM, Hartmann MC, Khan S, Boyle MP, Curran CE and Kerin MJ: Influence of stromal-epithelial interactions on breast cancer in vitro and in vivo. Breast Cancer Res Treat 131: 401-411, 2012.

12. O'Malley G, Treacy O, Lynch K, Naicker SD, Leonard NA, Lohan P, Dunne PD, Ritter T, Egan LJ and Ryan AE: Stromal cell PD-L1 inhibits $\mathrm{CD}^{+} \mathrm{T}$-cell antitumor immune responses and promotes colon cancer. Cancer Immunol Res 6: 1426-1441, 2018.

13. Trivanović D, Krstić J, Jauković A, Bugarski D and Santibanez JF Mesenchymal stromal cell engagement in cancer cell epithelial to mesenchymal transition. Dev Dyn 247: 359-367, 2018.
14. Patel SG and Shah JP: TNM staging of cancers of the head and neck: Striving for uniformity among diversity. CA Cancer J Clin 55: 242-258, 2005.

15. Spiro RH, Guillamondegui O Jr, Paulino AF and Huvos AG: Pattern of invasion and margin assessment in patients with oral tongue cancer. Head Neck 21: 408-413, 1999.

16. Hussein MR and Cullen K: Molecular biomarkers in HNSCC: Prognostic and therapeutic implications. Expert Rev Anticancer Ther 1: 116-24, 2001.

17. Takabatake K, Kawai H, Omori H, Qiusheng S, Oo MW, Sukegawa S, Nakano K, Tsujigiwa $\mathrm{H}$ and Nagatsuka H: Impact of the stroma on the biological characteristics of the parenchyma in oral squamous cell carcinoma. Int J Mol Sci 21: 7714, 2020.

18. Zhang X, Junior CR, Liu M, Li F, D'Silva NJ and Kirkwood KL: Oral squamous carcinoma cells secrete RANKL directly supporting osteolytic bone loss. Oral Oncol 49: 119-128, 2013.

19. An YZ, Cho E, Ling J and Zhang X: The Axin2-snail axis promotes bone invasion by activating cancer-associated fibroblasts in oral squamous cell carcinoma. BMC Cancer 20: 987, 2020.

20. Jögi A, Vaapil M, Johansson M and Påhlman S: Cancer cell differentiation heterogeneity and aggressive behavior in solid tumors. Ups J Med Sci 117: 217-224, 2012.

21. Watanabe M, Ohnishi Y, Wato M, Tanaka A and Kakudo K: SOX4 expression is closely associated with differentiation and lymph node metastasis in oral squamous cell carcinoma. Med Mol Morphol 47: 150-155, 2014.

22. Sun Z, Hu S, Luo Q, Ye D, Hu D and Chen F: Overexpression of SENP3 in oral squamous cell carcinoma and its association with differentiation. Oncol Rep 29: 1701-1706, 2013.

23. Kang MK, Chen W and Park NH: Regulation of epithelial cell proliferation, differentiation, and plasticity by grainyhead-like 2 during oral carcinogenesis. Crit Rev Oncog 23: 201-217, 2018.

24. Feng X, Zheng Z, Wang Y, Song G, Wang L, Zhang Z, Zhao J, Wang Q and Lun L: Elevated RUNX1 is a prognostic biomarker for human head and neck squamous cell carcinoma. Exp Biol Med (Maywood) 246: 538-546, 2021.

25. Gkouveris I, Nikitakis N, Avgoustidis D, Karanikou M, Rassidakis G and Sklavounou A: ERK1/2, JNK and STAT3 activation and correlation with tumor differentiation in oral SCC. Histol Histopathol 32: 1065-1076, 2017.

26. Sapkota D, Bruland O, Parajuli H, Osman TA, The MT, Johannessen AC and Costea DE: S100A16 promotes differentiation and contributes to a less aggressive tumor phenotype in oral squamous cell carcinoma. BMC Cancer 15: 631, 2015.

27. Kim B, Nam S, Lim JH and Lim JS: NDRG2 expression decreases tumor-induced osteoclast differentiation by down-regulating ICAM1 in breast cancer cells. Biomol Ther (Seoul) 24: 9-18, 2016.

28. Astarci E, Sade A, Cimen I, Savas B and Banerjee S: The NF- $\kappa$ B target genes ICAM-1 and VCAM-1 are differentially regulated during spontaneous differentiation of Caco-2 cells. FEBS J 279: 2966-2986, 2012.

29. Wang QL, Li BH, Liu B, Liu YB, Liu YP, Miao SB, Han Y, Wen JK and Han M: Polymorphisms of the ICAM-1 exon 6 (E469K) are associated with differentiation of colorectal cancer. J Exp Clin Cancer Res 28: 139, 2009.

30. Revu S, Wu J, Henkel M, Rittenhouse N, Menk A, Delgoffe GM, Poholek AC and McGeachy MJ: IL-23 and IL-1 $\beta$ drive human Th17 cell differentiation and metabolic reprogramming in absence of CD28 costimulation. Cell Rep 22: 2642-2653, 2018.

31. Xue G, Jin G, Fang J and Lu Y: IL-4 together with IL-1 $\beta$ induces antitumor Th9 cell differentiation in the absence of TGF- $\beta$ signaling. Nat Commun 10: 1376, 2019.

32. Albrecht M, Doroszewicz J, Gillen S, Gomes I, Wilhelm B, Stief T and Aumüller G: Proliferation of prostate cancer cells and activity of neutral endopeptidase is regulated by bombesin and IL-1beta with IL-1beta acting as a modulator of cellular differentiation. Prostate 58: 82-94, 2004.

33. Szemes M, Melegh Z, Bellamy J, Greenhough A, Kollareddy M, Catchpoole D and Malik K: A wnt-BMP4 signaling axis induces MSX and NOTCH proteins and promotes growth suppression and differentiation in neuroblastoma. Cells 9: 783, 2020.

34. Catalano V, Dentice M, Ambrosio R, Luongo C, Carollo R, Benfante A, Todaro M, Stassi G and Salvatore D: Activated thyroid hormone promotes differentiation and chemotherapeutic sensitization of colorectal cancer stem cells by regulating wnt and BMP4 signaling. Cancer Res 76: 1237-1244, 2016.

35. Zhang L, Sun H, Zhao F, Lu P, Ge C, Li H, Hou H, Yan M, Chen T, Jiang G, et al: BMP4 administration induces differentiation of $\mathrm{CD} 33^{+}$hepatic cancer stem cells, blocking their contributions to hepatocellular carcinoma. Cancer Res 72: 4276-4285, 2012. 
36. Fell SM,LiS, Wallis K, Kock A, Surova O,Rraklli V,Höfig CS,Li W, Mittag J, Henriksson MA, et al: Neuroblast differentiation during development and in neuroblastoma requires KIF1B $\beta$-mediated transport of TRKA. Genes Dev 31: 1036-1053, 2017.

37. Condello S, Caccamo D, Currò M, Ferlazzo N, Parisi G and Ientile R: Transglutaminase 2 and NF-kappaB interplay during NGF-induced differentiation of neuroblastoma cells. Brain Res 1207: 1-8, 2008.

38. Louhichi T, Saad H, Dhiab MB, Ziadi S and Trimeche M: Stromal CD10 expression in breast cancer correlates with tumor invasion and cancer stem cell phenotype. BMC Cancer 18: 49, 2018.

39. Moriyama T, Ohuchida K, Mizumoto K, Cui L, Ikenaga N, Sato $\mathrm{N}$ and Tanaka M: Enhanced cell migration and invasion of CD133+ pancreatic cancer cells cocultured with pancreatic stromal cells. Cancer 116: 3357-3368, 2010

40. Liu Y, Pan J, Pan X, Wu L, Bian J, Lin Z, Xue M, Su T, Lai S, Chen F, et al: Klotho-mediated targeting of CCL2 suppresses the induction of colorectal cancer progression by stromal cell senescent microenvironments. Mol Oncol 13: 2460-2475, 2019.

41. Kadaba R, Birke H, Wang J, Hooper S, Andl CD, Di Maggio F Soylu E, Ghallab M, Bor D, Froeling FE, et al: Imbalance of desmoplastic stromal cell numbers drives aggressive cancer processes. J Pathol 230: 107-117, 2013.

42. Sun LP, Xu K, Cui J, Yuan DY, Zou B, Li J, Liu JL, Li KY, Meng Z and Zhang B: Cancer-associated fibroblast-derived exosomal miR-382-5p promotes the migration and invasion of oral squamous cell carcinoma. Oncol Rep 42: 1319-1328, 2019.

43. Ding L, Ren J, Zhang D, Li Y, Huang X, Hu Q, Wang H, Song Y, Ni Y and Hou Y: A novel stromal lncRNA signature reprograms fibroblasts to promote the growth of oral squamous cell carcinoma via LncRNA-CAF/interleukin-33. Carcinogenesis 39: 397-406, 2018

44. Wang Y, Jing Y, Ding L, Zhang X, Song Y, Chen S, Zhao X, Huang $\mathrm{X}, \mathrm{Pu} \mathrm{Y}$, Wang $\mathrm{Z}$, et al: Epiregulin reprograms cancer-associated fibroblasts and facilitates oral squamous cell carcinoma invasion via JAK2-STAT3 pathway. J Exp Clin Cancer Res 38: 274, 2019.

45. Herrero AB, García-Gómez A, Garayoa M, Corchete LA, Hernández JM, San Miguel J and Gutierrez NC: Effects of IL-8 up-regulation on cell survival and osteoclastogenesis in multiple myeloma. Am J Pathol 186: 2171-2182, 2016.

46. Cioni B, Nevedomskaya E, Melis MH, van Burgsteden J, Stelloo S, Hodel E, Spinozzi D, de Jong J, van der Poel H, de Boer JP, et al: Loss of androgen receptor signaling in prostate cancer-associated fibroblasts (CAFs) promotes CCL2- and CXCL8-mediated cancer cell migration. Mol Oncol 12: 1308-1323, 2018.

47. New J, Arnold L, Ananth M, Alvi S, Thornton M, Werner L, Tawfik O, Dai H, Shnayder Y, Kakarala K, et al: Secretory autophagy in cancer-associated fibroblasts promotes head and neck cancer progression and offers a novel therapeutic target. Cancer Res 77: 6679-6691, 2017.

48. Giri J, Das R, Nylen E, Chinnadurai R and Galipeau J: CCL2 and CXCL12 derived from mesenchymal stromal cells cooperatively polarize IL-10+ tissue macrophages to mitigate gut injury. Cell Rep 30: 1923-1934.e4, 2020.

49. Yao M, Fang W, Smart C, Hu Q, Huang S, Alvarez N, Fields P and Cheng N: CCR2 chemokine receptors enhance growth and cell-cycle progression of breast cancer cells through SRC and PKC activation. Mol Cancer Res 17: 604-617, 2019.
50. Sun X, Glynn DJ, Hodson LJ, Huo C, Britt K, Thompson EW, Woolford L, Evdokiou A, Pollard JW, Robertson SA and Ingman WV: CCL2-driven inflammation increases mammary gland stromal density and cancer susceptibility in a transgenic mouse model. Breast Cancer Res 19: 4, 2017.

51. Tsuyada A, Chow A, Wu J, Somlo G, Chu P, Loera S, Luu T, Li AX, Wu X, Ye W, et al: CCL2 mediates cross-talk between cancer cells and stromal fibroblasts that regulates breast cancer stem cells. Cancer Res 72: 2768-2779, 2012.

52. Acharyya S, Oskarsson T, Vanharanta S, Malladi S, Kim J, Morris PG, Manova-Todorova K, Leversha M, Hogg N, Seshan VE, et al: A CXCL1 paracrine network links cancer chemoresistance and metastasis. Cell 150: 165-178, 2012.

53. Miyake M, Hori S, Morizawa Y, Tatsumi Y, Nakai Y, Anai S, Torimoto K, Aoki K, Tanaka N,Shimada K, et al:CXCL1-mediated interaction of cancer cells with tumor-associated macrophages and cancer-associated fibroblasts promotes tumor progression in human bladder cancer. Neoplasia 18: 636-646, 2016.

54. Park GY, Pathak HB, Godwin AK and Kwon Y: Epithelial-stromal communication via CXCL1-CXCR2 interaction stimulates growth of ovarian cancer cells through p38 activation. Cell Oncol (Dordr) 44: 77-92, 2021.

55. Zou A, Lambert D, Yeh H, Yasukawa K, Behbod F, Fan F and Cheng N: Elevated CXCL1 expression in breast cancer stroma predicts poor prognosis and is inversely associated with expression of TGF- $\beta$ signaling proteins. BMC Cancer 14: 781, 2014.

56. Wei LY, Lee JJ, Yeh CY, Yang CJ, Kok SH, Ko JY, Tsai FC and Chia JS: Reciprocal activation of cancer-associated fibroblasts and oral squamous carcinoma cells through CXCL1. Oral Oncol 88: 115-123, 2019.

57. Baba Y, Nosho K, Shima K, Meyerhardt JA, Chan AT, Engelman JA, Cantley LC, Loda M, Giovannucci E, Fuchs CS and Ogino S: Prognostic significance of AMP-activated protein kinase expression and modifying effect of MAPK3/1 in colorectal cancer. Br J Cancer 103: 1025-1033, 2010.

58. Lin J, Cao S, Wang Y, Hu Y, Liu H, Li J, Chen J, Li P, Liu J, Wang $Q$ and Zheng L: Long non-coding RNA UBE2CP3 enhances HCC cell secretion of VEGFA and promotes angiogenesis by activating ERK1/2/HIF-1 $\alpha$ /VEGFA signaling in hepatocellular carcinoma. J Exp Clin Cancer Res 37: 113, 2018.

59. YanZ, Ohuchida K,Fei S,Zheng B, Guan W, Feng H, Kibe S, Ando Y, Koikawa K, Abe T, et al: Inhibition of ERK1/2 in cancer-associated pancreatic stellate cells suppresses cancer-stromal interaction and metastasis. J Exp Clin Cancer Res 38: 221, 2019.

60. García-Carracedo D, Cai Y, Qiu W, Saeki K, Friedman RA, Lee A, Li Y, Goldberg EM, Stratikopoulos EE, Parsons R, et al: PIK3CA and p53 mutations promote 4NQO-initated head and neck tumor progression and metastasis in mice. Mol Cancer Res 18: 822-834, 2020.

61. Cohen Y, Goldenberg-Cohen N, Shalmon B, Shani T, Oren S, Amariglio N,Dratviman-Storobinsky O, Shnaiderman-Shapiro A, Yahalom R, Kaplan I and Hirshberg A: Mutational analysis of PTEN/PIK3CA/AKT pathway in oral squamous cell carcinoma. Oral Oncol 47: 946-950, 2011.

(i) 9 This work is licensed under a Creative Commons Attribution-NonCommercial-NoDerivatives 4.0 International (CC BY-NC-ND 4.0) License. 